\title{
SIMULATION OF THE FORMATION AND MORPHOLOGY OF ICE MANTLES ON INTERSTELLAR GRAINS
}

\author{
H. M. Cuppen ${ }^{1,2}$ and Eric Herbst ${ }^{1,3}$ \\ Received 2007 March 7; accepted 2007 June 8
}

\begin{abstract}
Although still poorly understood, the chemistry that occurs on the surfaces of interstellar dust particles profoundly affects the growth of molecules in the interstellar medium. An important set of surface reactions produces icy mantles of many monolayers in cold and dense regions. The monolayers are dominated by water ice, but also contain $\mathrm{CO}, \mathrm{CO}_{2}$, and occasionally methanol, as well as minor constituents. In this paper, the rate of production of water-ice-dominated mantles is calculated for different physical conditions of interstellar clouds and for the first time images of the morphology of interstellar ices are presented. For this purpose, the continuous-time random-walk Monte Carlo simulation technique has been used. The visual extinction, density, and gas and grain temperatures are varied. It is shown that our stochastic approach can reproduce the important observation that ice mantles only grow in the denser regions.
\end{abstract}

Subject headings: dust, extinction - ISM: abundances — ISM: molecules — stars: formation

Online material: color figures

\section{INTRODUCTION}

In cold and dense regions of interstellar clouds, infrared absorption studies show the existence of mantles of ices (Williams et al. 1992), of which water ice is observed to be the main constituent (Whittet et al. 1998; Pontoppidan et al. 2004). The formation and destruction of the interstellar ices is particularly important in regions where new stars and planets are born. During the first stage of star formation, small cores in a cloud assembly collapse under their own gravitational force, resulting in high gas densities and low temperatures. Under such conditions, virtually all gaseous species heavier than hydrogen and helium accrete onto the grains, where they can undergo chemical processes. In protostellar regions, the ices evaporate and change the nature of the gas-phase chemistry (Caselli et al. 1993; Bottinelli et al. 2004).

The formation of water-ice-dominated mantles is not yet fully understood. Recombination of smaller species on the surface and condensation of complete molecules from the gas phase are the competing processes, but quantitative estimates of their efficiency under interstellar conditions are lacking. Also, the reason for the apparent absence of water ice in more diffuse clouds, which are exposed to ultraviolet and visible photons, is still not clear. From observations of these regions, we know that the ice mantles constitute approximately less than a few monolayers of water, the current detection limit. A recent attempt to understand the formation of ice layers was made by Papoular (2005), who concluded that direct accretion of water from the gas is necessary to initiate ice-mantle formation. His main explanations for the lack of water ice in the diffuse regions are the high grain temperature and low flux in these regions.

As Papoular (2005), our goal in this paper is to explain the growth of monolayers of ice in dense sources and the absence of this growth in diffuse sources. With the use of a so-called microscopic Monte Carlo approach (see below), we focus on the accretive recombination mechanism using a set of surface reactions undergone by species that either accrete onto a grain surface or are

\footnotetext{
1 Department of Physics, Ohio State University, Columbus, OH 43210.

2 Leiden Observatory, Leiden University, P.O. Box 9513, 2300 RA Leiden, Netherlands.

3 Departments of Astronomy and Chemistry, The Ohio State University, Columbus, OH 43210.
}

products of other surface reactions that remain on the grain. The reactants are limited to species containing the elements $\mathrm{O}$ and $\mathrm{H}$. We also consider photodissociation processes for surface species caused by ultraviolet photons. In diffuse sources, these photons are mainly those of the external radiation field, whereas in dense sources, the much smaller flux of photons arises indirectly from cosmic-ray bombardment of $\mathrm{H}_{2}$, which produces ions and electrons. The electrons can then excite $\mathrm{H}_{2}$, leading to fluorescence (Prasad \& Tarafdar 1983; Ruffle \& Herbst 2001). In translucent and dense clouds, we find that ice mantles grow and we follow their morphology in some detail. To the best of our knowledge, this is an initial attempt to explain the morphology of ice grown via chemical reactions.

\section{MONTE CARLO METHOD}

The exact details of the continuous-time random-walk (CTRW) Monte Carlo method are already explained in previous papers (Chang et al. 2005; Cuppen \& Herbst 2005). We therefore give only a brief summary here. The initial grain surface is divided up into a square lattice of adsorption sites, each of which corresponds to a local energy minimum. We start from a bare surface of amorphous carbon with a high degree of roughness. Carbon is used rather than olivine because of the more severe lack of available desorption data for the adsorbates we must consider on the latter surface material. Since the major portion of carbonaceous grains is of an amorphous nature rather than crystalline, we treat the grain as being amorphous. This choice also means that the grains do not have a metallic character, as they would had they consisted of graphite. Figure 1 shows the topology of the surface of the bare carbonaceous grain we use throughout the paper. It is the same as surface (d) in Cuppen \& Herbst (2005) and surface III in Cuppen et al. (2006). Cuppen \& Herbst (2005) explain the procedure used to obtain this surface. The roughness is caused by a variety of surface imperfections, including vacancies (holes in the surface), edges, islands, and ad-atoms, all of which are shown in Figure 2 of Cuppen \& Herbst (2005). The species are confined to a square lattice structure even though we intend to simulate the growth of an amorphous ice mantle. An off-lattice simulation method, where the species are not confined to regular lattice positions, would seem to be a better choice, but this procedure is computationally expensive. Since we do not have a potential or force field to reliably 


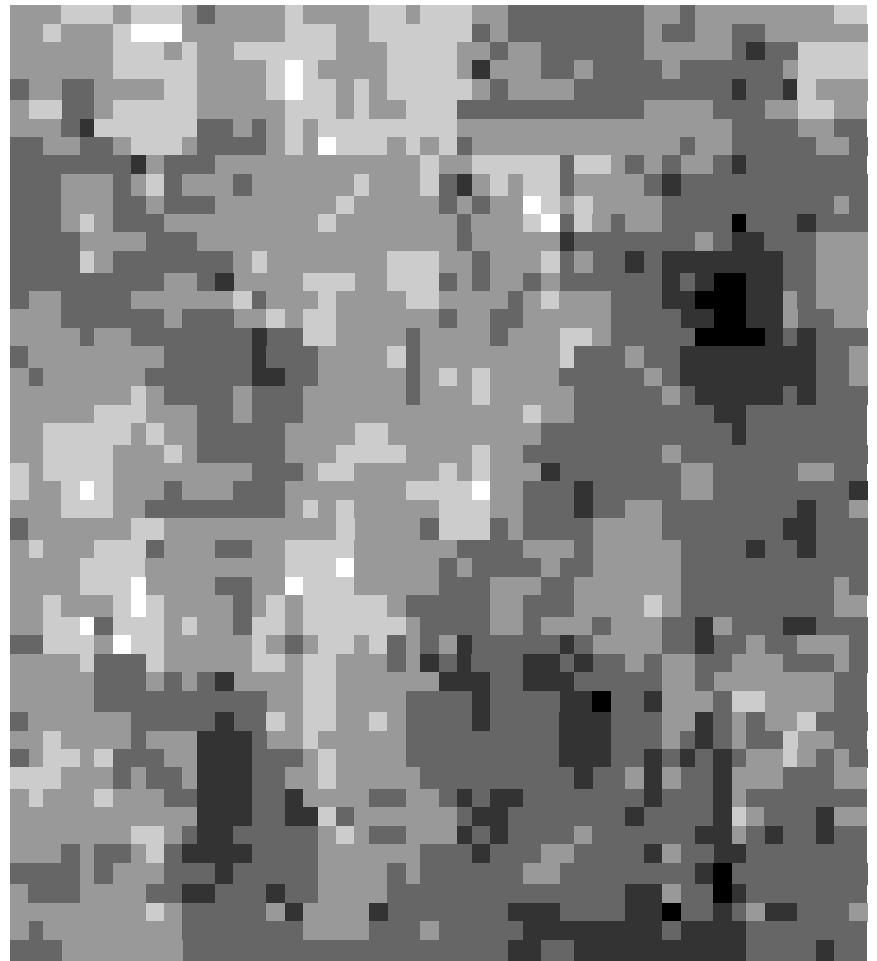

FIG. 1.-Surface topology of the bare carbonaceous grain, represented by a lattice of adsorption sites. The lighter colors indicate the higher areas. See Cuppen \& Herbst (2005) for a detailed discussion of how the surface was generated.

describe the system, it would not result in much more realistic simulations. The surface plotted in Figure 1 has $50 \times 50$ lattice sites. Because this size is above the limit where finite size effects play a role (Chang et al. 2005), scaling the results to larger sizes does not cause a problem. Indeed, we scale the results to a standard grain with a radius of $0.1 \mu \mathrm{m}$, which corresponds to a $250-$ 500 times larger grid, depending on the site density chosen. The smaller grid was used to keep the CPU time for the simulations reasonable.

Atoms of a species labeled A are deposited on these grains during our simulations with a deposition rate in monolayers (ML) $\mathrm{s}^{-1}$ of (Biham et al. 2001)

$$
R_{\mathrm{dep}}=\frac{v_{A} n_{\mathrm{A}}}{4 \rho}
$$

where $n_{\mathrm{A}}$ is the absolute gas abundance of species A, $\rho$ is the surface site density, and $v$ is the mean velocity of the atoms and molecules in the gas:

$$
v_{A}=\sqrt{\frac{8 k T_{\mathrm{gas}}}{\pi m_{A}}},
$$

with $T_{\text {gas }}$ the temperature of the gas and $m_{A}$, the atomic mass. We use $\rho=5 \times 10^{14} \mathrm{~cm}^{-2}$ in diffuse clouds and $\rho=1 \times 10^{15} \mathrm{~cm}^{-2}$ in dense clouds. The latter value is obtained from measurements of high-density ice (Jenniskens et al. 1995). The value used for diffuse clouds was determined for amorphous carbon (Biham et al. 2001). In our simulation, atoms hit the surface under a randomly determined angle. The atom will stick to some environment on the rough surface depending on its incoming angle. Once on the surface, the atom can hop over the surface, or desorb. The rates $\left(\mathrm{s}^{-1}\right)$ of hopping and desorption are, respectively,

$$
\begin{aligned}
& R_{\mathrm{hop}}=\nu_{b} \exp \left(-\frac{E_{b}}{T}\right), \\
& R_{\text {eva }}=\nu_{D} \exp \left(-\frac{E_{D}}{T}\right),
\end{aligned}
$$

where $T$ is the surface temperature of the grain, $E_{b}$ and $E_{D}$ are the hopping barrier and desorption energy $(\mathrm{K})$, and $\nu_{b}$ and $\nu_{D}$ are the attempt frequencies for hopping and desorption, respectively. The frequencies $\nu_{b}$ and $\nu_{D}$ are both taken to be $10^{12} \mathrm{~s}^{-1}$, which is a standard value for physisorbed species (Biham et al. 2001).

\subsection{Binding, Hopping, and Activation Energies}

We assume that species close to step edges or other protrusions of the surface are more strongly bound because of the extra lateral physisorption "bonds" with the surface. Only lateral interactions with the water ice and the bare carbonaceous grain are considered.

For the binding energy of a particular adsorbate species, labeled $\mathrm{A}$, we use the equation

$$
E_{D}^{\mathrm{A}}\left(i_{c}, i_{\mathrm{H}_{2} \mathrm{O}}\right)=E_{s}^{\mathrm{A}}+\alpha i_{c} E_{c}^{\mathrm{A}}+\alpha i_{\mathrm{H}_{2} \mathrm{O}} E_{\mathrm{H}_{2} \mathrm{O}}^{\mathrm{A}},
$$

where $E_{s}^{\mathrm{A}}$ is the binding energy of A with the particular surface lying beneath it, $E_{c}^{\mathrm{A}}$ is the binding energy of $\mathrm{A}$ with amorphous carbon, $E_{\mathrm{H}_{2} \mathrm{O}}^{\mathrm{A}}$ is the binding energy of A with water ice, $\alpha$ is the fraction of the binding energy that characterizes lateral binding, and $i_{s}\left(s=c, \mathrm{H}_{2} \mathrm{O}\right)$ is the number of horizontal neighbors of the particle with carbon or ice. For the hopping energies, we use the equation

$$
E_{b}^{\mathrm{A}}\left(i_{c}, i_{\mathrm{H}_{2} \mathrm{O}}\right)=0.5 E^{\mathrm{A}}+\alpha i_{c} E_{c}^{\mathrm{A}}+\alpha i_{\mathrm{H}_{2} \mathrm{O}} E_{\mathrm{H}_{2} \mathrm{O}}^{\mathrm{A}}
$$

which is similar to equation (5) except for the factor 0.5 in the first term. Very little is known about the relation between the hopping barrier and the desorption energy. For atomic hydrogen on amorphous carbon and polycrystalline silicate surfaces a factor between the hopping barrier and desorption energy of 0.78 was found (Katz et al. 1999). This value was obtained by fitting rate equations to temperature-programmed-desorption (TPD) experiments. In most astrochemical models, a much lower value of 0.3 is assumed (Tielens \& Allamandola 1987). Experiments of water-onwater diffusion indicate a similar value to 0.3 whereas $\mathrm{CO}$ on water ice experiments suggest a slightly higher ratio (Collings et al. 2003). Our value of 0.5 lies closer to this last study and corresponds to the value found by Ulbricht et al. (2002) for oxygen adsorption on carbon nanotubes. The higher value found by Katz et al. (1999) could be the result of an average over different hopping barriers. Because our method of lateral bonds already accounts for this effect, we take a lower value. Appendix A discusses the effect of this hopping factor on the results. Note that the lateral bonds must be broken in a hopping movement and that we do not account for the bonds that are reformed since we assume the barrier to be independent of these bonds. We do not consider a possible vertical interaction upwards for embedded layers. If a particle has "upper neighbors" we do not allow it to evaporate but it can hop, leaving a vacancy, or "pore," in the material.

The $\alpha$ factor is taken to be 0.2 in all simulations. Once again, very little is known about what this factor should be for physisorption. We choose our relatively low value to be conservative; nonzero values are needed to explain the high efficiency of $\mathrm{H}_{2}$ formation at relevant surface temperatures in diffuse clouds but 
TABLE 1

Surface Reactions

\begin{tabular}{|c|c|c|}
\hline Reaction & $\beta$ & $\begin{array}{c}E_{a} \\
(\mathrm{~K})\end{array}$ \\
\hline $\mathrm{H}+\mathrm{H} \rightarrow \mathrm{H}_{2} \ldots \ldots \ldots \ldots \ldots \ldots \ldots \ldots \ldots$ & $0.413^{\mathrm{a}}$ & 0 \\
\hline $\mathrm{H}+\mathrm{O} \rightarrow \mathrm{OH}$ & $0.991^{\mathrm{a}}$ & 0 \\
\hline $\mathrm{H}+\mathrm{OH} \rightarrow \mathrm{H}_{2} \mathrm{O}$ & $0.991^{\mathrm{a}}$ & 0 \\
\hline $\mathrm{O}+\mathrm{O} \rightarrow \mathrm{O}_{2}$ & $0.991^{\mathrm{a}}$ & 0 \\
\hline $\mathrm{H}+\mathrm{O}_{2} \rightarrow \mathrm{O}_{2} \mathrm{H}$ & $0.991^{\mathrm{a}}$ & $1200^{\mathrm{b}}$ \\
\hline $\mathrm{H}+\mathrm{O}_{2} \mathrm{H} \rightarrow \mathrm{H}_{2} \mathrm{O}_{2}$ & $0.991^{\mathrm{a}}$ & 0 \\
\hline $\mathrm{H}+\mathrm{H}_{2} \mathrm{O}_{2} \rightarrow \mathrm{H}_{2} \mathrm{O}+\mathrm{OH} \ldots \ldots \ldots \ldots$ & 1 & $1400^{\mathrm{c}}$ \\
\hline $\mathrm{H}+\mathrm{O}_{3} \rightarrow \mathrm{O}_{2}+\mathrm{OH}$ & 1 & $450^{\mathrm{d}}$ \\
\hline $\mathrm{H}_{2}+\mathrm{OH} \rightarrow \mathrm{H}_{2} \mathrm{O}+\mathrm{H}$ & 1 & $2600^{\mathrm{e}}$ \\
\hline $\mathrm{O}+\mathrm{O}_{2} \rightarrow \mathrm{O}_{3}$ & $0.991^{\mathrm{a}}$ & 0 \\
\hline
\end{tabular}

${ }^{\text {a }}$ Based on photodissociation of water results by Kroes \& Andersson (2006).

b Melius \& Blint (1979).

c Klemm et al. (1975).

${ }^{d}$ Lee et al. (1978)

e Schiff (1973).

high values can lead to complex TPD spectra in the laboratory with multiple peaks (Cuppen \& Herbst 2005). The factor has the largest effect on the hopping and desorption of hydrogen and on the hopping of oxygen atoms. All other species have such high energies for hopping and desorption that they remain in their sites independently of any changes in energies due to a different $\alpha$ factor.

If two particles encounter each other due to hopping and land in the same lattice site, a reaction can occur. Table 1 lists the surface reactions used to study ice formation, their estimated activation energies, and a factor $\beta$ that stands for the fraction of products remaining on the surface after a reaction. All of the surface reactions in Table 1 are exothermic. The excess energy that is released in an association reaction (a process with one product) can cause the product to desorb from the surface. To the best of our knowledge, $\beta$ has been determined experimentally only for the formation of $\mathrm{H}_{2}$ on olivine (0.33) and amorphous carbon (0.413) (Katz et al. 1999), but not on ice. The $\beta$-value for the formation of water from $\mathrm{H}$ and $\mathrm{OH}$ can be extracted from molecular dynamics simulations of the photodissociation of solid water performed by Kroes \& Andersson (2006). They find that a fraction of the $\mathrm{H}+\mathrm{OH}$ photodissociation products reacts back to water, $0.9 \%$ of which is released into the gas phase. We take this value of $\beta=0.991$ for all single-product association reactions. Only products in the outer layers, which are directly in contact with the gas, are allowed to evaporate. A value of $\beta$ near unity is also found in the theory of Garrod et al. (2006, 2007). This fraction is mainly important to remove water from the surface in diffuse clouds. It is of less importance for the $\mathrm{H}_{2}$ production, because $\mathrm{H}_{2}$ can evaporate thermally. Since the binding of molecular hydrogen to amorphous ices is similar to its binding to amorphous carbon, the $\beta$-value is expected to be similar on this substrate. We will use the value of $\beta=0.413$ for this reaction. A higher value will result in a slightly lower hydrogen recombination efficiency (Cuppen et al. 2006).

In contrast with might be expected from the case of gas-phase reactions, surface reactions with activation barriers can have noticeable contributions in our treatment, because the number of reaction trials the species can get before one of them hops away multiplied by the probability of reaction can reach unity (Awad et al. 2005). In our approach, if two species land in the same box and can react with activation energy, we do not follow the details of the competition but determine immediately which process-reaction or hopping-occurs by comparison of the two rates. There is experimental and/or theoretical evidence that these reactions do indeed occur on cold surfaces (Kroes \& Andersson 2006; Hiraoka et al. 1994; Tielens \& Hagen 1982; Watanabe \& Kouchi 2002). Here we assume that the reaction barrier can be treated as a rectangular potential barrier, through which tunneling occurs with a rate $\left(\mathrm{s}^{-1}\right)$

$$
R_{\text {react }}=\nu_{r} P_{\text {react }}=\nu_{r} \exp \left(-\frac{2 a}{h} \sqrt{\left(2 \mu E_{a}\right)}\right)
$$

where $a$ is the width of the barrier, $\mu$ the reduced mass, $\nu_{r}$ the attempt frequency for reaction, and $E_{a}$ the reaction activation energy. We assume that the trial frequency for hopping, desorption, and reaction is the same. We take here a width of $1 \AA$. The width of the diffusion barrier is probably larger (D. Woon 2007, private communication) and diffusion therefore most likely occurs through thermal hopping, as in the case of $\mathrm{H}_{2}$ formation (Katz et al. 1999). The difference in treatment between diffusion and hopping has a large influence on the temperature dependence of reactions with a barrier. The probability $P_{\text {react }}$ is temperature independent according to equation (7), whereas the thermal hopping rate, which is inversely proportional to the residence time, increases with increasing temperature. Tunneling leading to reaction therefore becomes more important as the temperature is lowered, but the decreased hopping rate makes it harder for two reactants to find each other. Detailed experiments and calculations need to be carried out to give more information about how to treat this type of surface reaction.

\subsection{Long-Range Diffusion and Photodissociation}

If a species tries to hop to a neighboring site that is already occupied and the two species do not react, we allow the particle X to diffuse to the next available free lattice site in the same layer with a probability $P$ given by the empirical equation

$$
P=\exp \left(-\frac{E_{D}\left(\mathrm{X} \text { on } \mathrm{H}_{2} \mathrm{O}\right)}{200} \Delta x\right),
$$

where $\Delta x$ is the distance in sites to the next available free site, and $E_{D}$ is the binding energy in $\mathrm{K}$. This equation is chosen so that small, weakly bound species such as atomic and molecular hydrogen can diffuse through porous ice structures, with a probability that rapidly decreases with increasing distance. Andersson et al. (2006) showed in molecular dynamics simulations that atomic hydrogen can move through the ice matrix after photodissociation of water, when the hydrogen atom is translationally excited. Equation (8) allows this motion to occur. The probability is an estimate based on the arguments mentioned above, since information for thermalized hydrogen at in the range of $10-20 \mathrm{~K}$ is lacking. Equation (8) does not contain an attempt frequency because this probability is only called if a normal hopping event cannot occur since the neighboring site is already occupied. The attempt frequency is therefore already in the hopping rate as is the temperature dependence. The particle can diffuse in all six directions. If the particle does not diffuse, it remains in its original position. Using binding energies discussed in the next section, the probability of diffusion of $\mathrm{H}$ over two sites $P$ is $4.8 \times 10^{-3}$, while it is $1.5 \times 10^{-8}$ for the two-site diffusion of a heavier ozone molecule. 
TABLE 2

Photodissociation Reactions

\begin{tabular}{|c|c|c|c|}
\hline Reaction $^{\mathrm{a}}$ & $\begin{array}{c}\alpha_{\text {photo }} \\
\left(\mathrm{s}^{-1}\right)\end{array}$ & $\gamma_{\text {photo }}$ & $\alpha_{\mathrm{cr}}$ \\
\hline $\mathrm{OH} \rightarrow \mathrm{O}+\mathrm{H}$ & $1.68(-10)$ & 1.66 & $1.02(3)$ \\
\hline $\mathrm{H}_{2} \mathrm{O} \rightarrow \mathrm{H}+\mathrm{OH} \ldots \ldots \ldots \ldots \ldots \ldots \ldots \ldots \ldots \ldots \ldots \ldots$ & $3.28(-10)$ & 1.63 & $1.94(3)$ \\
\hline $\mathrm{O}_{2} \rightarrow \mathrm{O}+\mathrm{O} \ldots \ldots \ldots \ldots \ldots \ldots \ldots \ldots \ldots \ldots \ldots \ldots \ldots \ldots \ldots \ldots$ & $3.30(-10)$ & 1.4 & $1.50(3)$ \\
\hline $\mathrm{O}_{2} \mathrm{H} \rightarrow \mathrm{O}+\mathrm{OH} \ldots \ldots \ldots \ldots \ldots \ldots \ldots \ldots \ldots \ldots \ldots$ & $\ldots$ & $\cdots$ & $1.50(3)$ \\
\hline $\mathrm{O}_{2} \mathrm{H} \rightarrow \mathrm{H}+\mathrm{O}_{2} \ldots \ldots \ldots \ldots \ldots \ldots \ldots \ldots \ldots \ldots \ldots \ldots$ & $\ldots$ & $\ldots$ & $1.50(3)$ \\
\hline $\mathrm{H}_{2} \mathrm{O}_{2} \rightarrow \mathrm{OH}+\mathrm{OH} \ldots \ldots \ldots \ldots \ldots \ldots \ldots$ & $\ldots$ & $\ldots$ & $3.00(3)$ \\
\hline $\mathrm{O}_{3} \rightarrow \mathrm{O}_{2}+\mathrm{O}$ & $1.9(-9)$ & $1.85^{\mathrm{b}}$ & $\ldots$ \\
\hline
\end{tabular}

${ }^{\text {a }}$ Gas-phase photodissociation rates and products are used.

${ }^{\mathrm{b}}$ Van Dishoeck et al. (2006).

Surface species can be destroyed by photodissociation, either directly or by cosmic-ray-induced photons. We assume that the photodissociation rate of the surface species is the same as in the gas phase, and is given by the standard expressions

$$
\begin{gathered}
R=\alpha_{\text {photo }} \exp \left(-\gamma_{\text {photo }} A_{V}\right), \\
R=\alpha_{\mathrm{CR}} \zeta,
\end{gathered}
$$

for direct and cosmic-ray-induced photodissociation, respectively. The constants are given in Table 2 and for the cosmic-ray ionization rate $\zeta$ we use $1.3 \times 10^{-17} \mathrm{~s}^{-1}$. These assumptions are only partially in agreement with molecular dynamics calculations for ice done by Kroes \& Andersson (2006), who show that the photodissociation cross section for water ice does not have exactly the same dependence on photon energy as is the case for gas-phase water. In our simulation, all molecules in the grain mantle can be photodissociated and a shielding mechanism is not included. Nguyen et al. (2002) assumed a limit of 100 monolayers that could be reached by photodissociation. In our simulations, only in dense regions where photodissociation plays a minor role can these thicknesses be reached.

We further assume that the heavy product fragments remain on the surface after photodissociation. These product fragments can, however, react, leading to the possibility of desorption in association reactions. Following Kroes \& Andersson (2006), we assume that $40 \%$ of the atomic hydrogen formed by photodissociation of $\mathrm{H}_{2} \mathrm{O}$ evaporates. This value is an average of the value they obtained for the first three layers. We also include their indirect mechanism for the photodesorption of water in which a thermally excited hydrogen atom kicks out one of the water molecules with an overall probability of $0.04 \%$.

\section{ENERGIES}

Table 3 contains the binding (desorption) energies used between assorted adsorbates and surfaces in our calculations. Many interaction energies are needed to describe the system, not just between the different species with the bare carbonaceous substrate but also with ice and with the other adsorbed species. The problem is, however, that many of these interaction energies are poorly known. For amorphous carbon, as far as we know, only the desorption energies for $\mathrm{H}$ and $\mathrm{H}_{2}$ are experimentally determined (Pirronello et al. 1999; Katz et al. 1999), with values of $658 \mathrm{~K}$ for $\mathrm{H}$ and $542 \mathrm{~K}$ for $\mathrm{H}_{2}$. Additional studies have been undertaken with graphite and carbon nanotubes. Even though our model grain consists of amorphous carbon, we occasionally have to rely on studies based on other forms of carbon. Ghio et al. (1980) calculated the van der Waals interaction between graphite and atomic hydrogen and found an energy of $503 \pm 6 \mathrm{~K}$, which is in excellent agreement with the overview of experimental data given by Vidali et al. (1991) in which the best estimate is given as $499 \pm 3 \mathrm{~K}$. For the $\mathrm{H}_{2}$ interactions with carbonaceous material we found two additional studies. Han \& Lee (2004) used DFT methods to calculate a value of $400 \mathrm{~K}$ for $\mathrm{H}_{2}$ on carbon nanotubes, while the survey of Vidali et al. (1991) gives a best estimate of $660 \pm 6 \mathrm{~K}$ for $\mathrm{H}_{2}$-graphite. In both cases, we use the amorphous carbon results.

For the other species, there are no amorphous carbon data and we have to make use of theoretical or experimental results for graphite, carbon nanotubes, or other forms of carbon. Papoular (2005) performed chemical simulations to obtain the interactions between $\mathrm{O}, \mathrm{OH}$, and $\mathrm{H}_{2} \mathrm{O}$ and typical hydrocarbon surface functional groups. He found energies of 300 and $500 \mathrm{~K}$ for $\mathrm{O}$ and $\mathrm{OH}$, respectively, while for water he found a range of energies between 250 and $1000 \mathrm{~K}$. The $\mathrm{O}$ and $\mathrm{OH}$ values seem rather low compared with the energies for the much lighter species $\mathrm{H}$ and $\mathrm{H}_{2}$. The calculations were performed between small single molecules and the absorbate. In reality, there will probably be many more contributions from other atoms in the substrate resulting in a higher interaction. We prefer to use the estimates of $800 \mathrm{~K}$ (Tielens \& Hagen 1982) for O and $1360 \mathrm{~K}$ (Allen \& Robinson 1975) for OH. For $\mathrm{H}_{2} \mathrm{O}$, there is another theoretical value by Picaud et al. (2004), who determined the energy to be $800 \mathrm{~K}$. Experimental results give 1870 K (Avgul \& Kiselev 1970) and 2150 K (Gale \& Beebe 1964) for the same system. We use $2000 \mathrm{~K}$ based on the experiments. For $\mathrm{O}_{2}$, we use the value of $1440 \mathrm{~K}$ from Ulbricht et al. (2002)

TABLE 3

\begin{tabular}{|c|c|c|c|c|c|c|c|c|c|c|}
\hline \multirow[b]{2}{*}{ Absorbate } & \multicolumn{10}{|c|}{ Substrate } \\
\hline & Carbon & $\mathrm{H}$ & $\mathrm{O}$ & $\mathrm{OH}$ & $\mathrm{H}_{2}$ & $\mathrm{O}_{2}$ & $\mathrm{H}_{2} \mathrm{O}$ & $\mathrm{O}_{3}$ & $\mathrm{O}_{2} \mathrm{H}$ & $\mathrm{H}_{2} \mathrm{O}_{2}$ \\
\hline 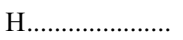 & 658 & $\ldots$ & $\ldots$ & $\ldots$ & 45 & 45 & 650 & 45 & $\ldots$ & 45 \\
\hline 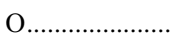 & 800 & $\ldots$ & $\ldots$ & 55 & 55 & 55 & 800 & 55 & 55 & 55 \\
\hline $\mathrm{OH}$ & 1360 & $\ldots$ & 240 & 240 & 240 & 240 & 3500 & 240 & 240 & 240 \\
\hline $\mathrm{H}_{2} \ldots \ldots \ldots \ldots \ldots \ldots \ldots$ & 542 & 30 & 30 & 30 & 23 & 30 & 440 & 30 & 30 & 30 \\
\hline $\mathrm{O}_{2} \ldots \ldots \ldots \ldots \ldots \ldots$ & 1440 & 69 & 69 & 69 & 69 & 69 & 1000 & 69 & 69 & 109 \\
\hline $\mathrm{H}_{2} \mathrm{O}$ & 2000 & 390 & 390 & 390 & 390 & 390 & 5640 & 390 & 390 & 390 \\
\hline $\mathrm{O}_{3} \ldots \ldots \ldots \ldots \ldots \ldots$ & 2240 & 120 & 120 & 120 & 120 & 120 & 1800 & 120 & 120 & 120 \\
\hline $\mathrm{O}_{2} \mathrm{H} \ldots \ldots \ldots \ldots \ldots$ & 2160 & $\ldots$ & 300 & 300 & 300 & 300 & 4300 & 300 & 300 & 300 \\
\hline $\mathrm{H}_{2} \mathrm{O}_{2} \ldots \ldots \ldots \ldots$ & 2818 & 340 & 340 & 340 & 340 & 340 & 4950 & 340 & 340 & 340 \\
\hline
\end{tabular}

Desorption Energies in Kelvins

Note.-See discussion in text. 
obtained by TPD experiments on graphite, while for the species $\mathrm{O}_{3}, \mathrm{O}_{2} \mathrm{H}$, and $\mathrm{H}_{2} \mathrm{O}_{2}$, we simply add the binding energies of their constituent parts:

$$
\begin{aligned}
E_{D}\left(\mathrm{O}_{3}\right) & =E_{D}\left(\mathrm{O}_{2}\right)+E_{D}(\mathrm{O}), \\
E_{D}\left(\mathrm{O}_{2} \mathrm{H}\right) & =E_{D}(\mathrm{OH})+E_{D}(\mathrm{O}), \\
E_{D}\left(\mathrm{H}_{2} \mathrm{O}_{2}\right) & =E_{D}\left(\mathrm{O}_{2} \mathrm{H}\right)+E_{D}(\mathrm{H}) .
\end{aligned}
$$

Let us next consider the energies of the species on solid water/ice. The problem here is that there are many types of ice, both in structure and in morphology and porosity, which makes it difficult to compare different experimental results. The porosity has two effects: (1) the particles in the pores cannot desorb directly but will perform a random walk on the wall of the pores until they reach the outer surface, and (2) the increased roughness in the pores means that the number of binding sites with high energy is probably larger. Since we already account for the latter effect by considering multiple types of sites, we use nonporous results as much as possible. A considerable amount of data is available for molecular hydrogen. Hornekær et al. (2005) and Dulieu et al. (2005) find a large distribution of energies using TPD experiments. If we only consider the mean value for the nonporous substrate we get $440 \mathrm{~K}$ from Hornekær et al. (2005) for $\mathrm{D}_{2}$ and 520 and $550 \mathrm{~K}$ from Dulieu et al. (2005) for $\mathrm{H}_{2}$ and $\mathrm{D}_{2}$, respectively. These values are close to the theoretical value obtained by Hollenbach \& Salpeter (1970) of $550 \mathrm{~K}$ and the experimental value of Sandford \& Allamandola (1993) of $550 \pm 35 \mathrm{~K}$, based on a mixture of water and methanol ice. The results of Perets et al. (2005), however, are in disagreement with this cluster of values. Performing TPD experiments for $\mathrm{HD}$ and $\mathrm{D}_{2}$ on high-density ice (HDI) and low-density ice (LDI), they found three types of sites for the LDI, which has a higher porosity and one type of site for the HDI with binding energies $798 \mathrm{~K}(\mathrm{HD})$ and $836 \mathrm{~K}\left(\mathrm{D}_{2}\right)$. We use $440 \mathrm{~K}$, the lower limit of the distribution and the peak energy found by Hornekær et al. (2005). Since we include higher energy sites near step edges in our model, we still obtain a distribution of desorption energies in this way.

For $\mathrm{H}$ on ice, we found three theoretical values: Hollenbach \& Salpeter (1970), Buch \& Czerminski (1991), and Al-Halabi et al. (2002) determined energies of $450 \mathrm{~K}, \approx 500 \mathrm{~K}$ and $400 \pm 50 \mathrm{~K}$, respectively. The values of Hollenbach \& Salpeter (1970) and Al-Halabi et al. (2002) are for crystalline ice, whereas the Buch \& Czerminski (1991) value is for amorphous ice. Perets et al. (2005) determined a significantly higher value, $720 \mathrm{~K}$, from TPD experiments on HDI. We use $650 \mathrm{~K}$, which is a new value calculated by Al-Halabi \& van Dishoeck (2007) for the binding energy of atomic hydrogen on amorphous ice. They found that the binding on amorphous ice is significantly higher than on crystalline ice due to the surface structure. Even a surface without protrusions of amorphous ice has several small cavities that will bind the hydrogen more strongly whereas the crystalline ice is even flat on an atomic scale.

For water on amorphous ice we use $5640 \mathrm{~K}$, which is the result obtained by Speedy et al. (1996) and is in excellent agreement with a value of $\approx 5600 \mathrm{~K}$ found by Fraser et al. (2001). For $\mathrm{O}_{2}$ we use $1000 \mathrm{~K}$, which is an average value obtained from the TPD data by Ayotte et al. (2001) and Collings et al. (2004). For O and $\mathrm{OH}$ we again take the estimates from Tielens \& Hagen (1982) and Allen \& Robinson (1975), respectively, and for $\mathrm{O}_{3}, \mathrm{O}_{2} \mathrm{H}$, and $\mathrm{H}_{2} \mathrm{O}_{2}$, we again add the binding energies of their constituent parts.

Let us now consider $\mathrm{H}_{2}$ as a substrate, since, as we will see, its abundance in the mantles can be considerable. Vidali et al. (1991) give an estimate of the desorption energy for $\mathrm{H}$ from $\mathrm{H}_{2}$ of $35.4 \pm 5.8 \mathrm{~K}+k T$ which leads to $45.4 \mathrm{~K}$ at $10 \mathrm{~K}$. The rest of the desorption energies from $\mathrm{H}_{2}$ are, starting from the ice desorption energies, scaled according to the equation

$$
E_{D}\left(\mathrm{X} \text { on } \mathrm{H}_{2}\right)=\frac{E_{D}\left(\mathrm{X} \text { on } \mathrm{H}_{2} \mathrm{O}\right)}{E_{D}\left(\mathrm{H} \text { on } \mathrm{H}_{2} \mathrm{O}\right)} E_{D}\left(\mathrm{H} \text { on } \mathrm{H}_{2}\right) \text {. }
$$

Once again, energies involving $\mathrm{O}_{3}, \mathrm{O}_{2} \mathrm{H}$, and $\mathrm{H}_{2} \mathrm{O}_{2}$ are formed from their fragments.

The reason the desorption energy of $\mathrm{O}_{2} \mathrm{H}$ is considered to be the sum of the desorption energies of $\mathrm{OH}$ and $\mathrm{O}$ instead of $\mathrm{O}_{2}$ and $\mathrm{H}$, which are better known, is the capability of $\mathrm{O}_{2} \mathrm{H}$ to form $\mathrm{H}$-type bonds. We are aware that desorption energies obtained in this manner have a large uncertainty. Fortunately, our simulations show the uncertainties in the desorption energies of the strongly bound species to have very little effect on the final result, since these species will remain on the surface at these temperatures within the timescale of the simulation regardless of their exact desorption energies.

\section{RESULTS}

In all simulations the water was formed through surface reactions and not by direct accretion from the gas phase, even though gas-phase water can be detected in some dense objects. This exclusion is easily justified. A typical value obtained in gas-phase models for the fractional abundance of water is at most $10^{-6}$. For a cloud of density $10^{4} \mathrm{~cm}^{-3}$, and a standard site density, we then calculate that the flux rate for gaseous water at $10 \mathrm{~K}$ on a grain is $3 \times 10^{-14} \mathrm{ML} \mathrm{s}^{-1}$, so that it takes roughly $10^{6} \mathrm{yr}$ to deposit a single monolayer and the enormous time of $10^{8} \mathrm{yr}$ to deposit the 100 monolayers typically associated with dense cloud mantles. The timescales are much longer for diffuse clouds. The flux of oxygen atoms arriving on grains is much higher than the flux of water molecules, typically by a factor of 100 in dense clouds. With a high efficiency of conversion to water by surface reactions, 100 monolayers of water can then be produced in $10^{6} \mathrm{yr}$, in reasonable agreement with our dense-cloud results discussed below. So, achieving significant mantle abundances in reasonable times does require surface reactions.

\subsection{Diffuse and Translucent Clouds}

We have performed a set of simulations for different values of the visual extinction $A_{V}$, total hydrogen density $\left(n_{\mathrm{H}}\right)$, and gas and grain temperatures. Figure 2 plots the surface abundances in monolayers for the different species in our model as functions of time. In this figure, the results for diffuse and translucent clouds are contained. All panels are labeled with a letter followed by a number. The panels starting with the same letter are grouped in rows and have the same visual extinction, which increases from top to bottom. The digits along a row indicate the different physical conditions. For the three leftward panels, the density is fixed but the temperatures decrease as one goes from left to right, while the rightmost panel (e.g., A4) has the same temperature as the second of the three left panels (e.g., A2) but a higher density. The exact physical conditions for each of the simulations are given in Table 4. In all cases shown in Figure 2, we assume the cloud gas to be initially atomic, with an oxygen abundance of $3.0 \times 10^{-4} n_{\mathrm{H}}$ that of hydrogen (Nguyen et al. 2002). The calculations are run for sufficiently short times that the gas-phase abundances remain unchanged. In all cases we start with a bare carbonaceous grain.

Figure 2 clearly shows that in diffuse regions $\left(A_{V}=0.5,1\right.$, rows $\mathrm{A}$ and $\mathrm{B}$ ) less than $1 \mathrm{ML}$ of water ice is present at a time of 


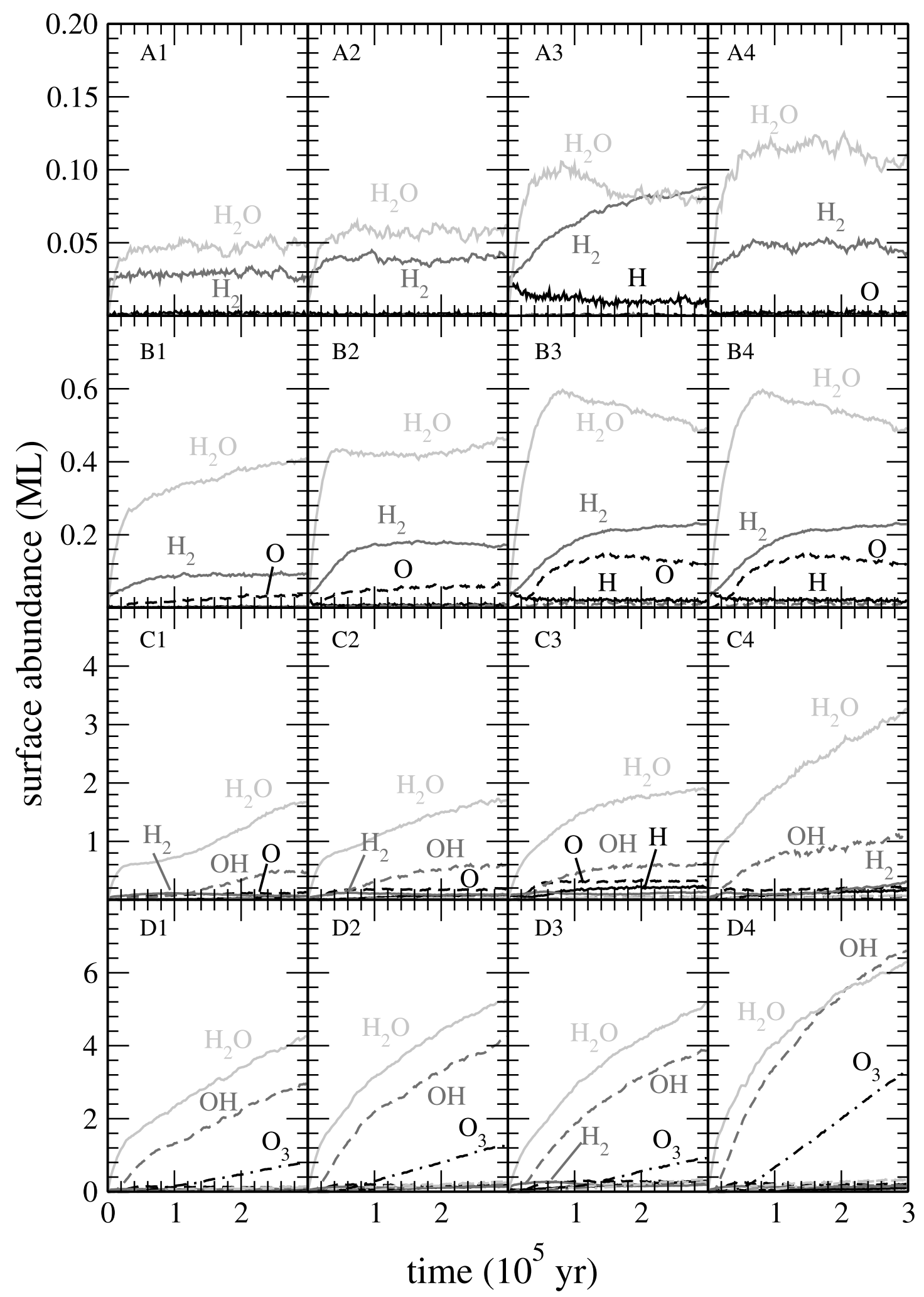

FIG. 2.-Surface abundance as a function of time for different conditions in a diffuse or translucent molecular cloud. See Table 4 for physical conditions in each panel. [See the electronic edition of the Journal for a color version of this figure.]

$3 \times 10^{5} \mathrm{yr}$, the largest time studied. For panels A1-A3, the water ice abundance has reached steady state conditions, while in panel $\mathrm{A} 4$, which refers to a higher density, steady state seems to be reached around the end of the simulation. In panels B1 and B2 the water layer is still growing, albeit slowly, while in panels B3 and B4 the water abundance is decreasing. Although water ice is not destroyed chemically in our model, a steady state results when destruction by photons and desorption from the mantle balance formation. Water ice is the most abundant species for the diffuse clouds in rows A and B; nonetheless, other species can be seen in the panels. Despite its low desorption energy, molecular hydrogen can reach mantle abundances of a significant fraction 
TABLE 4

Physical Conditions of Chosen Models

\begin{tabular}{|c|c|c|c|c|c|}
\hline Panel & $A_{V}$ & $\begin{array}{l}T_{\text {grain }} \\
(\mathrm{K})\end{array}$ & $\begin{array}{l}T_{\text {gas }} \\
(\mathrm{K})\end{array}$ & $\begin{array}{c}n_{\mathrm{H}} \\
\left(\mathrm{cm}^{-3}\right)\end{array}$ & Form of $\mathrm{H}$ \\
\hline ...…….... & 0.5 & 20 & 100 & $1.0(2)$ & $\mathrm{H}$ \\
\hline $\mathrm{A} 2 \ldots \ldots \ldots \ldots \ldots$ & 0.5 & 18 & 80 & $1.0(2)$ & $\mathrm{H}$ \\
\hline A3 & 0.5 & 16 & 60 & $1.0(2)$ & $\mathrm{H}$ \\
\hline A4 ..................... & 0.5 & 18 & 80 & $5.0(2)$ & $\mathrm{H}$ \\
\hline B1 ....................... & 1.0 & 18 & 80 & $2.5(2)$ & $\mathrm{H}$ \\
\hline 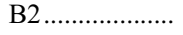 & 1.0 & 16 & 60 & $2.5(2)$ & $\mathrm{H}$ \\
\hline 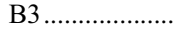 & 1.0 & 14 & 40 & $2.5(2)$ & $\mathrm{H}$ \\
\hline 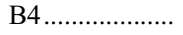 & 1.0 & 16 & 60 & $5.0(2)$ & $\mathrm{H}$ \\
\hline $\mathrm{C} 1$........................ & 2.0 & 17 & 70 & $5.0(2)$ & $\mathrm{H}$ \\
\hline $\mathrm{C} 2 \ldots \ldots \ldots \ldots$ & 2.0 & 15 & 50 & $5.0(2)$ & $\mathrm{H}$ \\
\hline 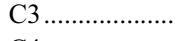 & 2.0 & 13 & 30 & $5.0(2)$ & $\mathrm{H}$ \\
\hline $\mathrm{C} 4 \ldots \ldots \ldots \ldots \ldots \ldots$ & 2.0 & 15 & 50 & $1.0(3)$ & $\mathrm{H}$ \\
\hline 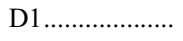 & 3.0 & 16 & 60 & $1.0(3)$ & $\mathrm{H}$ \\
\hline 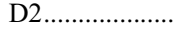 & 3.0 & 14 & 40 & $1.0(3)$ & $\mathrm{H}$ \\
\hline 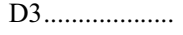 & 3.0 & 12 & 20 & $1.0(3)$ & $\mathrm{H}$ \\
\hline D4 & 3.0 & 14 & 40 & $1.5(3)$ & $\mathrm{H}$ \\
\hline E1 ....................... & 5.0 & 14 & 40 & $5.0(3)$ & $\mathrm{H}_{2}$ \\
\hline E2 …..................... & 5.0 & 12 & 20 & $5.0(3)$ & $\mathrm{H}_{2}$ \\
\hline E3 ….................... & 5.0 & 10 & 10 & $5.0(3)$ & $\mathrm{H}_{2}$ \\
\hline E4 ....................... & 5.0 & 12 & 20 & $1.0(4)$ & $\mathrm{H}_{2}$ \\
\hline F1 ...................... & 10.0 & 12 & 20 & $2.0(3)$ & $\mathrm{H}_{2}$ \\
\hline 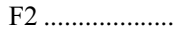 & 10.0 & 10 & 10 & $2.0(4)$ & $\mathrm{H}_{2}$ \\
\hline F3 & 10.0 & 10 & 10 & $1.0(4)$ & $\mathrm{H}_{2}$ \\
\hline F4 ..................... & 10.0 & 10 & 10 & $5.0(4)$ & $\mathrm{H}_{2}$ \\
\hline
\end{tabular}

Note.-The notation a(b) implies $a \times 10^{b}$.

of a monolayer in diffuse clouds. In the translucent clouds, rows $\mathrm{C}$ and $\mathrm{D}$, the water ice abundance exceeds $1 \mathrm{ML}$ by the end of $3 \times 10^{5} \mathrm{yr}$ and is still growing. Water ice is dominant in all panels except $\mathrm{D} 4$, where $\mathrm{OH}$ has a comparable total abundance. As far as we know, no data from observations is available about the abundance of $\mathrm{OH}$ present on grains under comparable conditions.

If we look at the influence of the physical conditions on the formation of ice for diffuse and translucent clouds, it can be concluded that the gas and grain temperature affect the ice formation mechanism only to a small extent. In particular, a low surface tem- perature results in a slightly thicker ice mantle. In general, the ice thickness is mostly determined by photodissociation under the influence of the radiation field and by the gas density. Thus, as one goes down a column and to the rightmost panel of any row (higher density), the water ice abundance increases. Figure 2 shows a clear difference in the composition of the ice layer depending on the visual extinction. At $A_{V}=0.5$ (A panels) the mantle mainly consists of water, and molecular hydrogen. For increasing $A_{V}$ the amounts of $\mathrm{OH}$ and $\mathrm{O}_{3}$ increase. The surface abundance of atomic oxygen appears to peak at $A_{V}=2$ (C panels).

Figure 3 shows a vertical slice of the ice at $3 \times 10^{5} \mathrm{yr}$ for four different conditions - panels A2, B2, C2, and D2-in which visual extinction and gas density increase. The different molecules are color coded in the panels, while gray represents the carbon substrate and black represents the void. One sees immediately that the mantle for A2 is virtually nonexistent, while that for D2 has grown to a significant number of monolayers. The mantles are certainly porous; there is a lot of black interspersed among the color-coded molecules. Due to the strong radiation in all simulations, the ice goes through many cycles of formation and destruction. Let us take a more detailed look at mantles $\mathrm{C} 2$ and D2. The top layers of the mantles are primarily water (white color). As one goes deeper into the mantle, one notices more green $(\mathrm{OH})$, and finally toward the bottom one finds lots of magenta (ozone). In understanding this dependence of abundance on mantle height, the topology of the mantle is important. Blocking of the evaporation of the hydroxyl radicals and oxygen atoms after photodissociation and obstruction of the deposition of fresh hydrogen from the gas phase are the main reasons that allow the large abundances of $\mathrm{OH}$ and $\mathrm{O}_{3}$ to be formed away from the surface. Atomic hydrogen leaves the surface either by direct desorption after photodissociation or in the form of $\mathrm{H}_{2}$ that can diffuse through the pores. We used the same desorption probability of $\mathrm{H}$ after water photodissociation of 0.4 throughout the ice. If $\mathrm{H}$ atoms accreting from the gas have a greater penetration depth or if a depth dependence of the desorption probability on photodissociation was considered, the large abundance variance with depth we predict could be partially reduced. Unfortunately, very little experimental information on the structure of water ices formed through surface reactions under interstellar conditions is available so that our images of the ice mantles cannot be easily compared with real ices.
A2

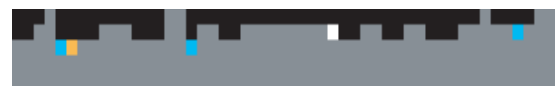

C2

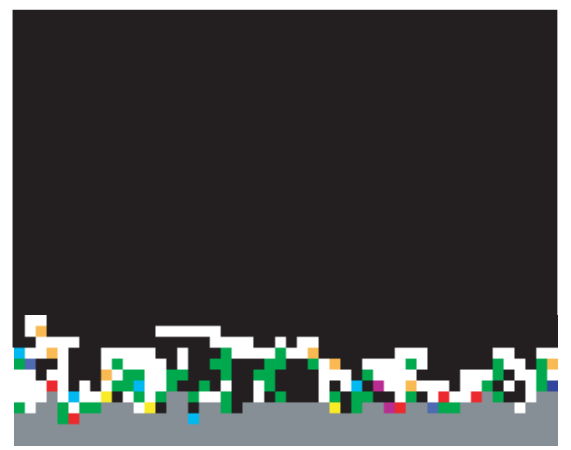

B2

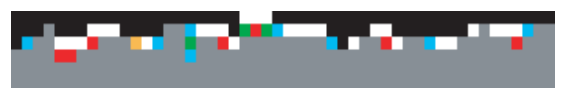

D2

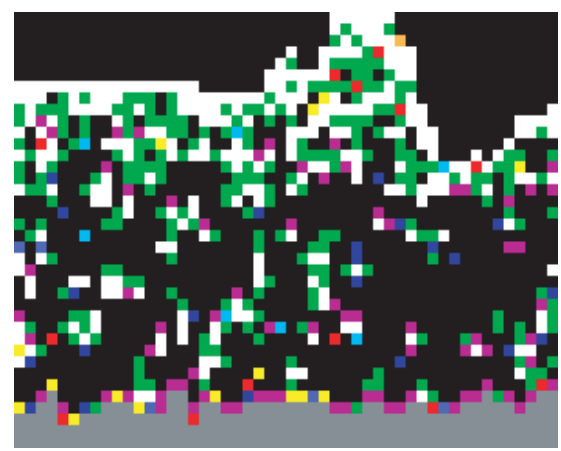

grain

void

0

H

$\mathrm{O}_{2}$

$\mathrm{OH}$

$\mathrm{H}_{2}$

$\mathrm{O}_{2} \mathrm{H}$

$\mathrm{H}_{2} \mathrm{O}_{2}$

$\mathrm{O}_{3}$

$\mathrm{H}_{2} \mathrm{O}$

Fig. 3.-Vertical cross sections of the ice mantles after $3 \times 10^{5}$ yr for four different physical conditions in diffuse and translucent clouds (A2, B2, C2, D2; Table 4). The molecules are color coded. 
TABLE 5

$\mathrm{H}_{2}$ Recombination Efficiency

\begin{tabular}{|c|c|c|c|c|c|c|c|c|}
\hline \multirow[b]{2}{*}{ Panel } & \multicolumn{4}{|c|}{ Oxygen Flux Present } & \multicolumn{4}{|c|}{ Oxygen Flux Absent } \\
\hline & 1 & 2 & 3 & 4 & 1 & 2 & 3 & 4 \\
\hline 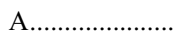 & 0.05 & 0.04 & 0.22 & 0.05 & 0.02 & 0.17 & 0.57 & 0.11 \\
\hline $\mathrm{B}$ & 0.06 & 0.15 & 0.24 & 0.17 & 0.28 & 0.59 & 0.72 & 0.60 \\
\hline $\mathrm{C}$ & 0.24 & 0.29 & 0.48 & 0.22 & 0.54 & 0.65 & 0.71 & 0.68 \\
\hline $\mathrm{D}$ & 0.11 & 0.43 & 0.52 & 0.54 & 0.61 & 0.74 & 0.71 & 0.73 \\
\hline
\end{tabular}

The common gas-grain codes (Ruffle \& Herbst 2001) do not keep track of the molecular structure of the ice layers so structural variations cannot be found. The mean field approach of most of these methods allows all species to react with each other independently of their position. The mantle abundances of the hydroxyl radical and ozone will therefore be lower in these simulations using the same surface reaction network since their reactions with $\mathrm{H}$ atoms will occur more readily. Even in our variegated ices, the mantle abundances of $\mathrm{OH}$ and $\mathrm{O}_{3}$ may decrease to some extent if other reactive species like $\mathrm{CO}$ are included and are allowed to react with $\mathrm{OH}$ and $\mathrm{O}_{3}$ in our reaction network. For the latter case these reactive species must be able to diffuse in the lower layers of the ice.

Molecular hydrogen formation from the recombination of two hydrogen atoms on a surface is the most important reaction in the diffuse interstellar medium. The recombination efficiency for $\mathrm{H}_{2}$ formation is defined by the ratio of twice the hydrogen molecules formed and leaving the surface divided by the flux of hydrogen atoms. This efficiency has been determined over the time interval that steady state is achieved for the 16 sets of diffuse and translucent physical conditions (A-D) given in Table 4 and the results are given in Table 5 by the left value in each column. The values on the right indicate the efficiency for the same densities and temperatures but without oxygen in the system, which means without ice being formed. There is a clear difference in efficiency, with the ice surface being less efficient in most cases. This is due to two effects: the first is that the hydrogen atoms are also used to form water, but this is only of minor importance. The larger effect is caused by the changing energetics between the water layers and the carbonaceous substrate. In particular, the binding energy of $\mathrm{H}$ on an ice surface is slightly less than on a carbonaceous grain and also the surface roughness changes due to the build-up of the water layers. In these calculations, we only use an $\alpha$ of 0.2 for the lateral bond strength. A higher $\alpha$ would result in a more efficient molecular hydrogen production because it causes the hydrogen atoms to be more strongly bound and to reside longer on the surface (Cuppen \& Herbst 2005). The efficiencies for lower surface temperatures $(12-15 \mathrm{~K})$ are generally still high enough to produce a reasonable amount of $\mathrm{H}_{2}$ (Cuppen et al. 2006) but the efficiencies are clearly degraded at higher temperatures. For smaller grains, we expect stochastic heating to reduce the formation of ice since both oxygen and hydrogen atoms will usually evaporate when a photon hits a grain. This effect would cause the recombination efficiency for molecular hydrogen to approach those reported in Cuppen et al. (2006).

\subsection{Dense Molecular Clouds}

Figure 4 is similar to Figure 2 but shows results for higher visual extinctions $\left(A_{\mathrm{V}}=5,10\right)$ and densities $\left(5.0 \times 10^{3}\right.$ to $\left.5.0 \times 10^{4} \mathrm{~cm}^{-3}\right)$. The maximum time considered is $1 \times 10^{5} \mathrm{yr}$, at which time steady state abundances have not yet been reached for the major constituents. Again the visual extinction is varied vertically while temperature and density for a fixed extinction are varied horizontally. The exact conditions are once again given in Table 4 . We assume that the hydrogen in the gas phase is mostly molecular. A relative abundance of $1 \times 10^{-4} n_{\mathrm{H}}$ for atomic $\mathrm{H}$ is used. Although it is assumed that the mantles grow on a carbonaceous substrate, it is more likely that they grow on ice mantles of the type discussed in the previous section for translucent clouds. It can be seen in Figure 4 that in seven out of the eight panels, the growth of water ice dominates the mantles, while in one panel (F4) the abundance of $\mathrm{H}_{2}$ overtakes that of water at later times. Mantles of up to 7580 monolayers are developed.

In the panels, a general tendency can be observed that a lower temperature (compare E1, E2, and E3) and a lower density (compare E2 and E4) result in less ice. This is in contrast with the results in Figure 2, where the amount of ice increases for decreasing surface temperature. In the diffuse clouds the grain temperature is in the regime where $\mathrm{H}$ atoms easily evaporate. Reducing the surface temperature will therefore result in more hydrogen on the surface increasing the efficiency of the hydrogenation reactions. The lower grain temperature in the dense clouds lies in the regime where the desorption of hydrogen molecules starts to slow down and these molecules occupy a large part of the mantle, slowing down the rate of hydrogenation reactions. Although laboratory simulations of interstellar ice reactions show that hydrogenation reactions are still possible in the presence of $\mathrm{H}_{2}$ because the hydrogen can still penetrate $\mathrm{H}_{2}$ layers (Fuchs et al. 2007), the reactions are slowed down, which results in an optimum temperature for the reaction rate. For $\mathrm{CO}+\mathrm{H}$, this optimum lies between 12 and $15 \mathrm{~K}$ (Fuchs et al. 2007).

Since the atomic hydrogen abundance is much lower than the molecular hydrogen abundance, the main route to form water ice changes in dense clouds. Table 6 gives the contributions of the three reactions that form water: $\mathrm{H}+\mathrm{OH}, \mathrm{H}+\mathrm{H}_{2} \mathrm{O}_{2}$, and $\mathrm{OH}+\mathrm{H}_{2}$. This table clearly indicates that the main route for formation is through the association of $\mathrm{H}$ and $\mathrm{OH}$ in the diffuse and translucent clouds. Since $\mathrm{H}$ is abundant in the gas here and since this reaction is barrierless, this mechanism is the most apparent one. However in dense clouds, the reaction between $\mathrm{H}_{2}$ and $\mathrm{OH}$ becomes the most common route and also the reaction of $\mathrm{H}$ with $\mathrm{H}_{2} \mathrm{O}_{2}$ makes a significant contribution. It seems surprising that in a dense molecular cloud a reaction with an activation barrier is so important for the formation of water ice. This is different from the gas-grain network simulations where the barrierless reaction remains the main route (Ruffle \& Herbst 2001) along with some other surface reactions that were not included in this work. The difference is mainly due to the difference in treatment of the activation barriers, as explained earlier: because of our approach, in which the competition with diffusion is modeled directly, reactions 


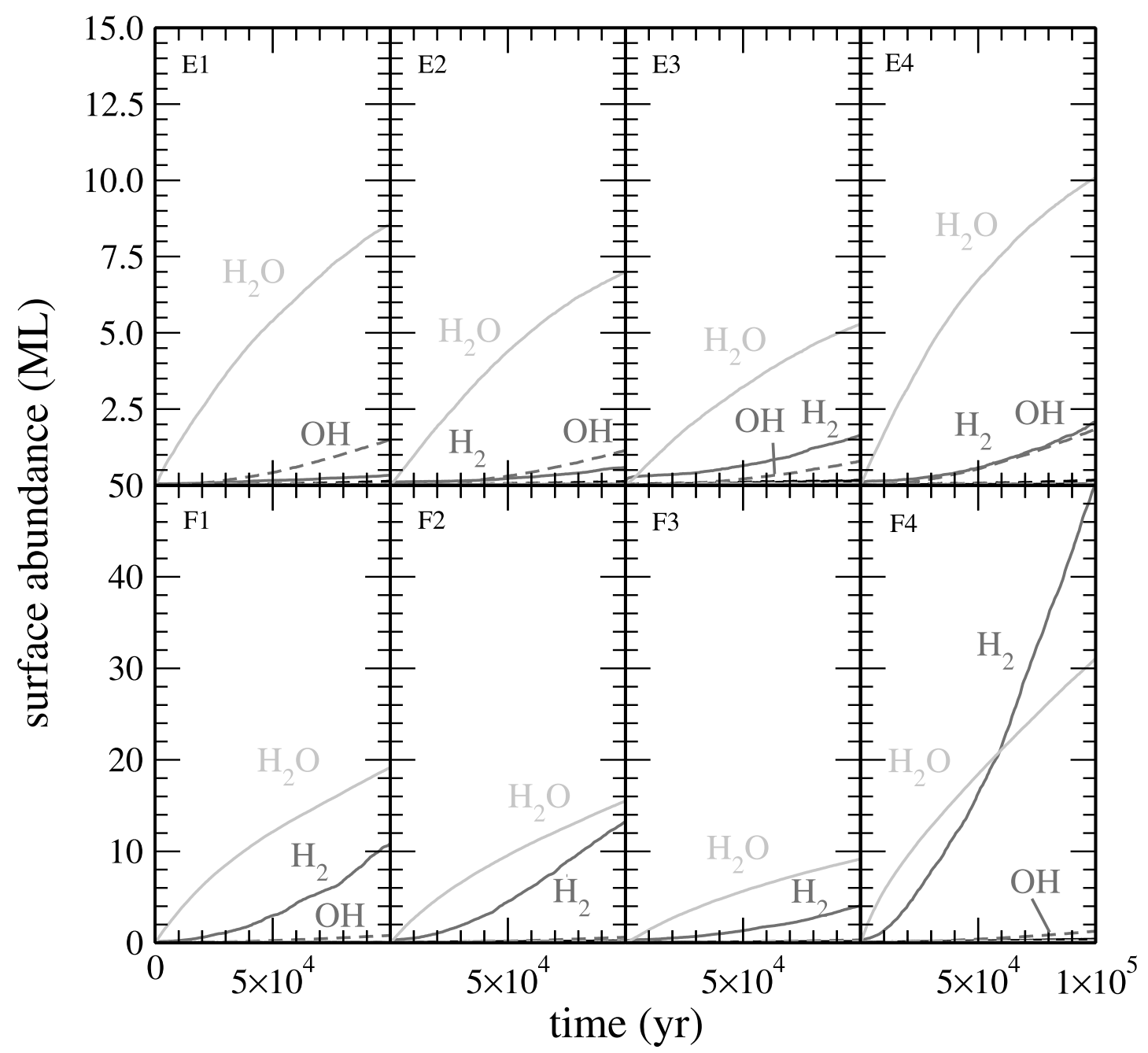

Fig. 4.-Surface abundance as a function of time for different conditions in a dense molecular cloud. The notation a(b) implies $a \times 10^{b}$. See Table 4 for physical conditions in each panel. [See the electronic edition of the Journal for a color version of this figure.]

with a barrier can have enhanced rates. In the approach used in the models, tunneling under the activation energy barrier is simply treated as a factor in the expression for the rate coefficient.

Figure 5 gives two vertical snapshots of ice mantles. Again the surface structure is very rough and porous. Unlike the diffuse cloud ices, however, the structures demonstrate a skyscraper, or protrusion, effect, which is even more pronounced for lower diffusion rates, as discussed in Appendix A. Since the protrusions contain a significant amount of molecular hydrogen and since we have not seen them up to now, it is likely that they can only be associated with cold dense cloud conditions with the ratio of diffusion barrier to desorption energy of 0.5 as is used here. The width and height of the protrusions are associated with the random angle deposition method. With this method, the protrusions continue to grow since they prevent incoming atoms from reaching the surface via a shadowing effect. It is likely that the tendency to form such noticeable skyscraper structures will be mitigated in the interstellar medium for several reasons. First, the grain surfaces are curved, which reduces the shadowing effect. A standard grain with a radius of $0.1 \mu \mathrm{m}$ has a circumference of approximately 2000 sites, which means the snapshots in Figure 5 represent arcs of $9^{\circ}$. For a small grain of $r=0.02 \mu \mathrm{m}$, it corresponds to $45^{\circ}$. Second, the rate of diffusion considered in our models is likely a lower limit because diffusion also occurs in the bulk structures that are formed. These will result in a smoother surface with less molecular hydrogen (see the following paragraph). To confirm our hypothesis concerning the random angle deposition method, we also performed simulations with perpendicular deposition. Large single protrusions containing a high percentage of $\mathrm{H}_{2}$ were not obtained under these conditions; only structures similar to the left panel in Figure 5 were found. The sticking fraction (fraction remaining in the mantles) in the simulation with the perpendicular deposition is much higher and a thicker layer of ice is formed. Angular deposition gives more irregular structures at the surface that have a weaker binding energy and can therefore desorb more easily, resulting in a lower sticking fraction.

Our results can profitably be compared with another simulation. Kimmel et al. (2001) studied the porosity of ice mantles formed by deposition of water molecules using a ballistic algorithm. In their algorithm they deposited the molecules under specific angles to simulate a molecular beam or with varying angles to simulate a background pressure. One of their conclusions is that ices deposited from a background pressure are very rough and porous, in agreement with our result that random angle deposition results in such structures. Kimmel et al. (2001) also let a molecule anneal immediately after its deposition. Their annealing algorithm consists of several cycles in which the deposited species hop to energetically more favored sites. Annealing results in denser ices (fewer narrow skyscrapers). We do not include annealing in our simulations, but we do include diffusion of $\mathrm{H}$ and $\mathrm{O}$ atoms 
TABLE 6

Contributions of Three Reactions to Water Formation

\begin{tabular}{|c|c|c|c|c|c|c|c|c|c|c|c|c|}
\hline \multirow[b]{2}{*}{ Panel } & \multicolumn{4}{|c|}{$\mathrm{H}+\mathrm{OH} \rightarrow \mathrm{H}_{2} \mathrm{O}$} & \multicolumn{4}{|c|}{$\mathrm{H}+\mathrm{H}_{2} \mathrm{O}_{2} \rightarrow \mathrm{H}_{2} \mathrm{O}+\mathrm{OH}$} & \multicolumn{4}{|c|}{$\mathrm{H}_{2}+\mathrm{OH} \rightarrow \mathrm{H}_{2} \mathrm{O}+\mathrm{H}$} \\
\hline & 1 & 2 & 3 & 4 & 1 & 2 & 3 & 4 & 1 & 2 & 3 & 4 \\
\hline А & 99.9 & 99.6 & 95.9 & 99.5 & 0.0 & 0.0 & 0.0 & 0.0 & 0.1 & 0.4 & 4.2 & 0.5 \\
\hline В & 99.5 & 98.3 & 96.4 & 98.5 & 0.0 & 0.0 & 0.0 & 0.1 & 0.5 & 1.7 & 3.6 & 1.4 \\
\hline С & 98.4 & 97.1 & 93.1 & 95.9 & 0.5 & 0.8 & 1.0 & 1.3 & 1.1 & 2.0 & 5.9 & 2.9 \\
\hline $\mathrm{D}$ & 97.6 & 93.4 & 90.5 & 93.2 & 1.4 & 1.4 & 1.6 & 0.9 & 1.0 & 5.2 & 7.9 & 5.9 \\
\hline 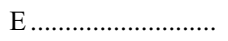 & 14.3 & 11.6 & 7.0 & 9.7 & 21.0 & 19.3 & 16.7 & 18.3 & 64.7 & 69.1 & 76.3 & 72.0 \\
\hline $\mathrm{F}$ & 10.1 & 6.5 & 6.2 & 6.7 & 18.7 & 16.7 & 16.5 & 16.2 & 71.2 & 76.8 & 77.2 & 77.1 \\
\hline
\end{tabular}

whereas the water molecules of Kimmel et al. (2001) do not diffuse thermally. In agreement with the observations of Kimmel et al. (2001) is our result that more diffusion results in denser ices, as can be seen by comparing the broad skyscrapers in Figure 5 with the narrow ones discussed in Appendix A.

\section{DISCUSSION}

Using the CTRW Monte Carlo method, we have shown that mantles of water and other ices can grow efficiently in cold and dense regions of the interstellar medium, whereas only a small amount, in the submonolayer regime, is formed under the conditions of diffuse interstellar clouds. Ices in translucent sources occupy an intermediate regime. We have also looked carefully at the morphology of the ices. This initial exploration is, however, dependent on a variety of poorly constrained parameters such as barriers to diffusion, which are generally less well understood than adsorption energies. With our standard model, the ices appear to be much denser in diffuse and translucent sources, although there are many pores in the interior. Also, there is a vertical stratification in these ices, in which water ice dominates in the uppermost monolayers while unusual species such as $\mathrm{OH}$ and $\mathrm{O}_{3}$ are abundant in the lower monolayers. In the dense sources, the ices, which are dominated by water and occasionally $\mathrm{H}_{2}$, appear to develop a skyscraper-type structure, which is somewhat dependent on the rate of diffusion adopted. This very rough structure is probably exaggerated somewhat in our calculations because we do not properly include diffusion inside the bulk. Although the water ice in the diffuse and translucent clouds is produced by the association reaction between $\mathrm{H}$ atoms and $\mathrm{OH}$ radicals, which is customarily assumed to be the dominant source of water, the situation in the dense clouds, which contain low abundances of gasphase hydrogen atoms, is quite different.

Observations in dense cloud assemblies such as Taurus show no water ice below a threshold value of $A_{V}=3.2$ and linear growth of the column density of water ice above this value (Whittet et al. 2001). In particular, with a least-squares fit, the expression

$$
N\left(\mathrm{H}_{2} \mathrm{O}\right)=q\left(A_{V}^{\mathrm{obs}}-A_{\mathrm{th}}\right)
$$

can be determined (Whittet et al. 2001), where $q=(1.30 \pm 0.04 \times$ $\left.10^{17}\right)_{\mathrm{cm}^{-2}}$ and $A_{\text {th }}=3.2 \pm 0.1$, using the relationship

$$
N\left(\mathrm{H}_{2} \mathrm{O}\right)=1.8 \times 10^{18}\left(\mathrm{~cm}^{-2}\right) \tau_{3.0}
$$

Relating these observations to the results of our simulations is not completely straightforward. The measured threshold value of $A_{V}=3.2$ is observed through the cloud and gives an edge-to-edge value. The observed ice content is the integrated contribution along a line of sight that typically contains heterogeneous material. The visual extinction experienced by most material along this line is lower, on average one-half of the edge-to-edge value.
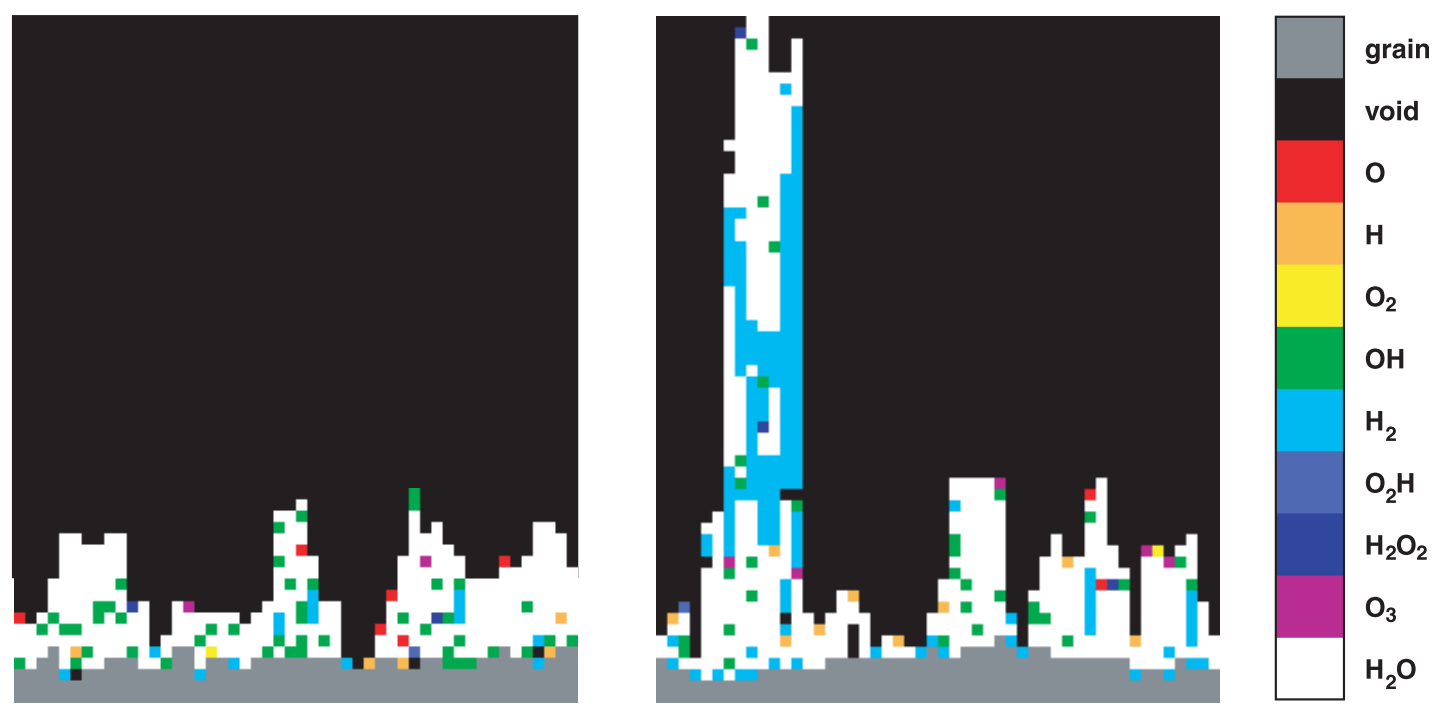

FIG. 5. - Vertical cross sections of the ice mantles after $1 \times 10^{5}$ yr for two different dense-cloud physical conditions. Left panel $(E 2): A_{V}=5, T_{\mathrm{gr}}=12 \mathrm{~K}, T_{\mathrm{gas}}=20 \mathrm{~K}$, and $n_{\mathrm{H}}=5 \times 10^{3} \mathrm{~cm}^{-3}$. Right panel (F2): $A_{V}=10, T_{\mathrm{gr}}=10 \mathrm{~K}, T_{\mathrm{gas}}=10 \mathrm{~K}$, and $n_{\mathrm{H}}=2 \times 10^{4} \mathrm{~cm}^{-3}$. The molecules are color coded. 


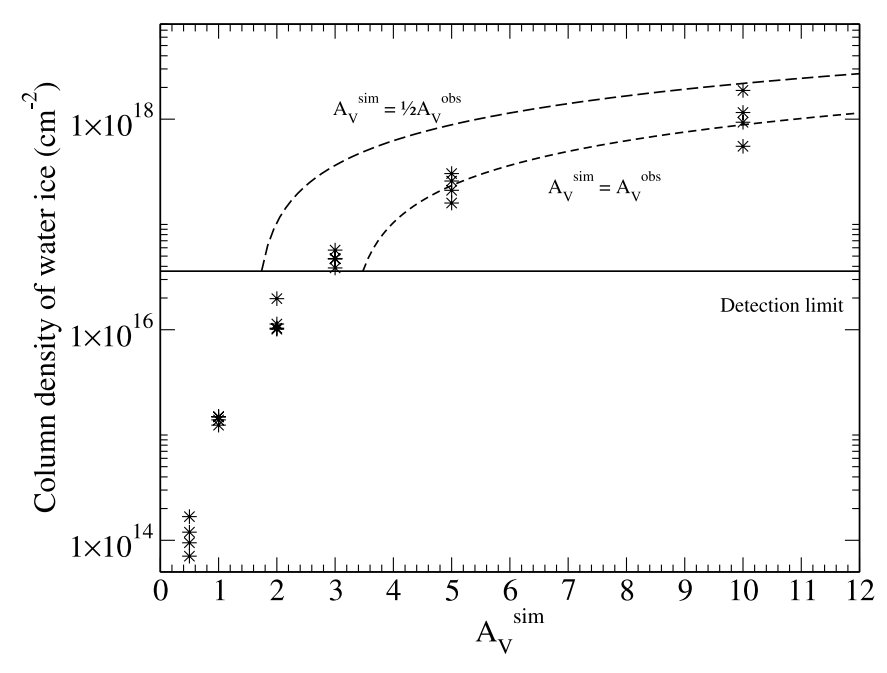

FIG. 6.-Column density of water ice at the end of the simulations $\left(3 \times 10^{5} \mathrm{yr}\right.$ for $A_{V}^{\text {sim }}=0.5, \ldots, 3$ and $10^{5}$ yr for $A_{V}^{\text {sim }}=5$ and 10) vs. the extinction used in our simulations for all physical conditions given in Figs. 2-4.

We make the assumption that the extinction along any line of sight is dominated by one object, defined by the physical conditions used for our models and listed in Table 4. The number of monolayers at $10^{5} \mathrm{yr}$ for the dense objects and $3 \times 10^{5} \mathrm{yr}$ for the diffuse and translucent objects is converted into column densities of ice using the gas-to-dust ratio for standard-sized grains and typical cloud depths (Nguyen et al. 2002). The results are plotted in Figure 6 at the six different visual extinctions used in our simulations. The several different results at each extinction correspond to the different physical conditions chosen. Also depicted in the figure are two dashed lines that represent the results of equation (15), assuming that the observed edge-to-edge extinctions correspond to our extinctions $-A_{V}^{\mathrm{sim}}=A_{V}^{\mathrm{obs}}$-and that they correspond to twice our extinctions: $A_{V}^{\operatorname{sim}}=\frac{1}{2} A_{V}^{\text {obs }}$. Finally, there is a solid line representing roughly the lower detectable limit for water ice based on the $\mathrm{H}_{2} \mathrm{O}$ optical depth at $3 \mu \mathrm{m}$ toward Cyg OB 2 No. 12 of $\tau<0.02$ (Whittet et al. 1997). This optical depth corresponds to an $N\left(\mathrm{H}_{2} \mathrm{O}\right)$ of $3.6 \times 10^{16} \mathrm{~cm}^{-2}$, via equation (16), which is on the order of a few monolayers. Assuming that the typical extinction seen by ice along a line of sight is indeed half the edge-to-edge value, our results should approach the upper dashed line shown in Figure 6. The plot indicates, however, that our simulations produce less ice than needed by a factor of $\approx 3-10$.

This shortfall can be accounted for by increasing the depth of our sources and, for dense clouds, extending the simulations to later times since the calculated ice abundances are still increasing rapidly at our latest time of $10^{5} \mathrm{yr}$. However, cosmic-ray desorption starts to become important after $10^{5} \mathrm{yr}$ and part of the grain mantle can desorb in this way (Herbst \& Cuppen 2006). It may be that we underestimate the efficiency of water ice formation, especially at intermediate values of the visual extinction. If so, we can produce more ice if the rates of diffusion are enhanced, as discussed in Appendix A. Also a decrease of the shadowing effect due to the presence of curvature, as discussed earlier, can result in thicker ice layers on small grains as we saw in the perpendicular deposition simulations. Yet another possibility is that some ice is indeed accreted from gaseous water, possibly in the cold gas following a shock, which would allow water to be produced in the gas via the reaction between $\mathrm{OH}$ and $\mathrm{H}_{2}$, despite its activation energy.

E. H. would like to thank the National Science Foundation (US) for supporting his research program in astrochemistry.

\section{APPENDIX A}

\section{THE INFLUENCE OF THE HOPPING BARRIERS}

A ratio of 0.5 between the diffusion barrier and desorption energy has been assumed throughout the paper. This appendix shows the simulation results using an alternative ratio of 0.78 , generalizing the result obtained by Katz et al. (1999) for atomic hydrogen on olivine.

The results are shown in Figures 7-10 and in Table 7. Figure 7 shows surface abundances for diffuse and translucent cloud conditions as a function of time, while Figure 8 does the same for dense cloud conditions. Figure 9 contains vertical cross sections of ice under diffuse and translucent conditions, while Figure 10 contains such images for dense cloud conditions. Table 7 lists $\mathrm{H}_{2}$ recombination efficiencies for diffuse and translucent clouds. As can be seen by comparing these figures and table with the analogous ones for our standard hopping barrier, there are important differences in the amount of ice, the recombination efficiency for molecular hydrogen formation, and in the structure of the ice between these results and the previous ones. Let us consider them in turn.

Considering the structure of the mantles, the ice is less dense for the higher hopping barrier, as can be clearly seen by comparing Figures 3 and 9 and Figures 5 and 10. This can be explained by realizing that for the lower hopping barrier, oxygen atoms can diffuse to more favorable positions (with more neighbors) before reaction.

Large protrusions as the one shown in the left panel of Figure 10 can be observed for both diffusion ratios, but they are more common and higher for the higher ratio, and are even present in translucent sources. The main reason is that the mobility of species like $\mathrm{O}$, $\mathrm{H}$, and $\mathrm{H}_{2}$ is limited to such an extent that they cannot move to an energetically more favorable position before it is covered by another particle. In cold dense clouds this effect is the strongest since the surface temperature is low and the flux of, especially, molecular hydrogen is very high. As explained in the main text, the formation of these protrusions is amplified by self-shadowing of the surface by the protrusion. Since a real interstellar grain surface will have a curvature, which becomes significant for smaller grains of approximately $0.02 \mu \mathrm{m}$, this shadowing effect will be less in reality.

More ice (see Fig. 7 in comparison with Fig. 2) and more molecular hydrogen are formed for the higher ratio. This is consistent with previous results for molecular hydrogen formation. If we compare the right columns in Tables 5 and 7 , we see that also in the case where only a hydrogen flux is present, the production of molecular hydrogen is much higher. 


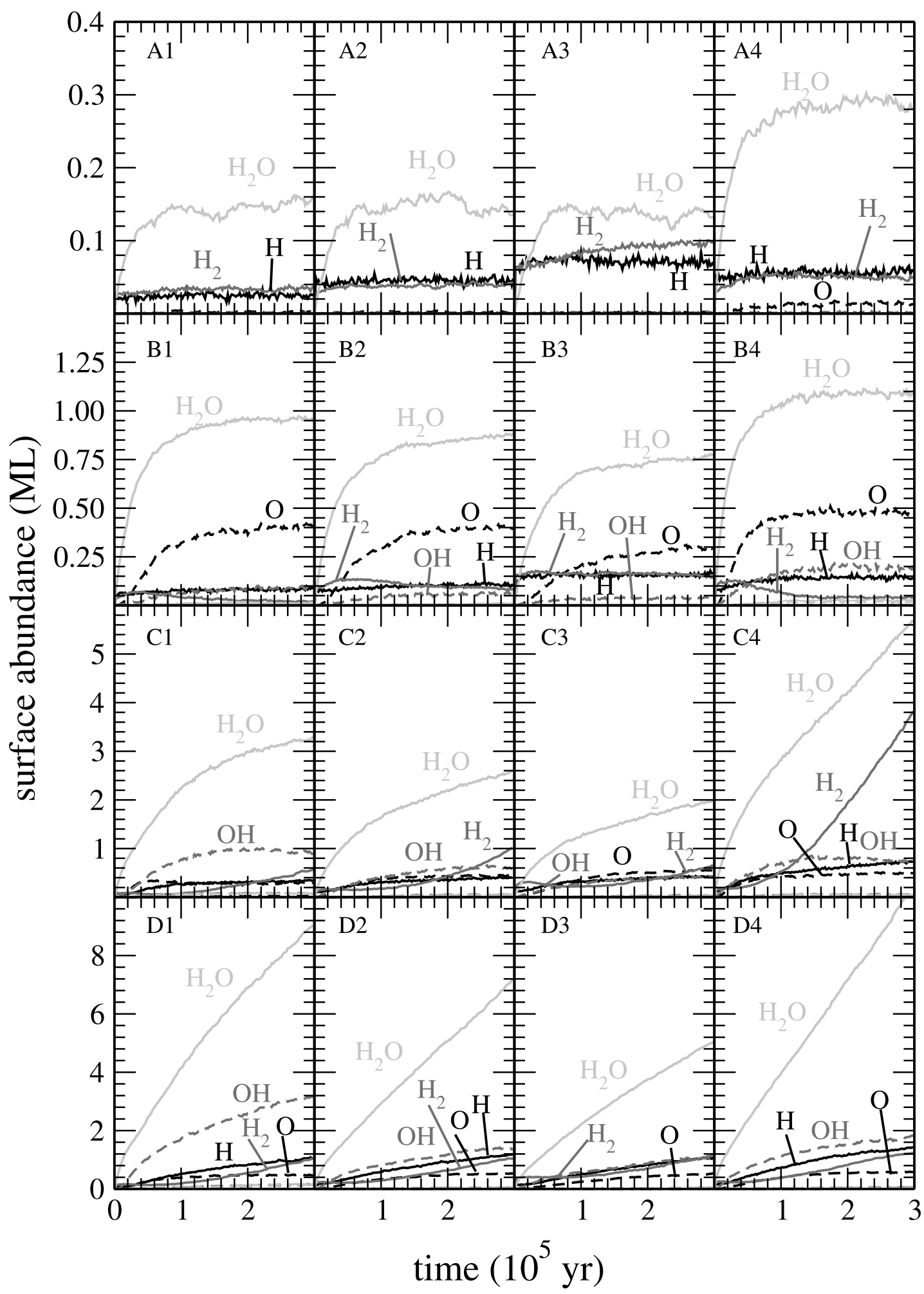

FIG. 7.- Surface abundance with a high hopping barrier-to-desorption energy of 0.78 as a function of time for different conditions in a diffuse or translucent molecular cloud. [See the electronic edition of the Journal for a color version of this figure.] 
TABLE 7

$\mathrm{H}_{2}$ Recombination with Slow Diffusion

\begin{tabular}{|c|c|c|c|c|c|c|c|c|}
\hline \multirow[b]{2}{*}{ Panel } & \multicolumn{4}{|c|}{ Oxygen Flux Present } & \multicolumn{4}{|c|}{ Oxygen Flux Absent } \\
\hline & 1 & 2 & 3 & 4 & 1 & 2 & 3 & 4 \\
\hline А & 0.47 & 0.58 & 0.61 & 0.59 & 0.56 & 0.72 & 0.68 & 0.69 \\
\hline В & 0.67 & 0.64 & 0.68 & 0.67 & 0.74 & 0.67 & 0.71 & 0.68 \\
\hline $\mathrm{C}$ & 0.40 & 0.43 & 0.49 & 0.37 & 0.71 & 0.74 & 0.65 & 0.75 \\
\hline $\mathrm{D}$ & 0.30 & 0.34 & 0.32 & 0.31 & 0.70 & 0.68 & 0.69 & 0.67 \\
\hline
\end{tabular}

Notes.-The left columns are results in the presence of an oxygen flux, the right in its absence. The calculations are done with a ratio of 0.78 between hopping barrier and desorption energy.

\section{APPENDIX B}

\section{THE INFLUENCE OF THE ROUGHNESS OF THE GRAIN}

This Appendix contains the results of simulations starting with a less rough grain than our standard one. With this smoother surface, the running time of the simulations becomes significantly longer. This effect occurs because on the smoother surface the average hopping time is smaller so that the simulation program spends more of its time evaluating hopping events. For this reason we performed simulations only for a few of the conditions in Table 4. Surface abundances versus time are shown in Figure 11 for panels A3, $\mathrm{B} 3, \mathrm{C} 3$, and D3.

In general, there is little difference between these results and our standard ones. The amount of ice that is formed is very similar for all simulations, except for the initial peak around $5 \times 10^{4} \mathrm{yr}$ for B3 when the smoother surface is used. The surface abundances of the

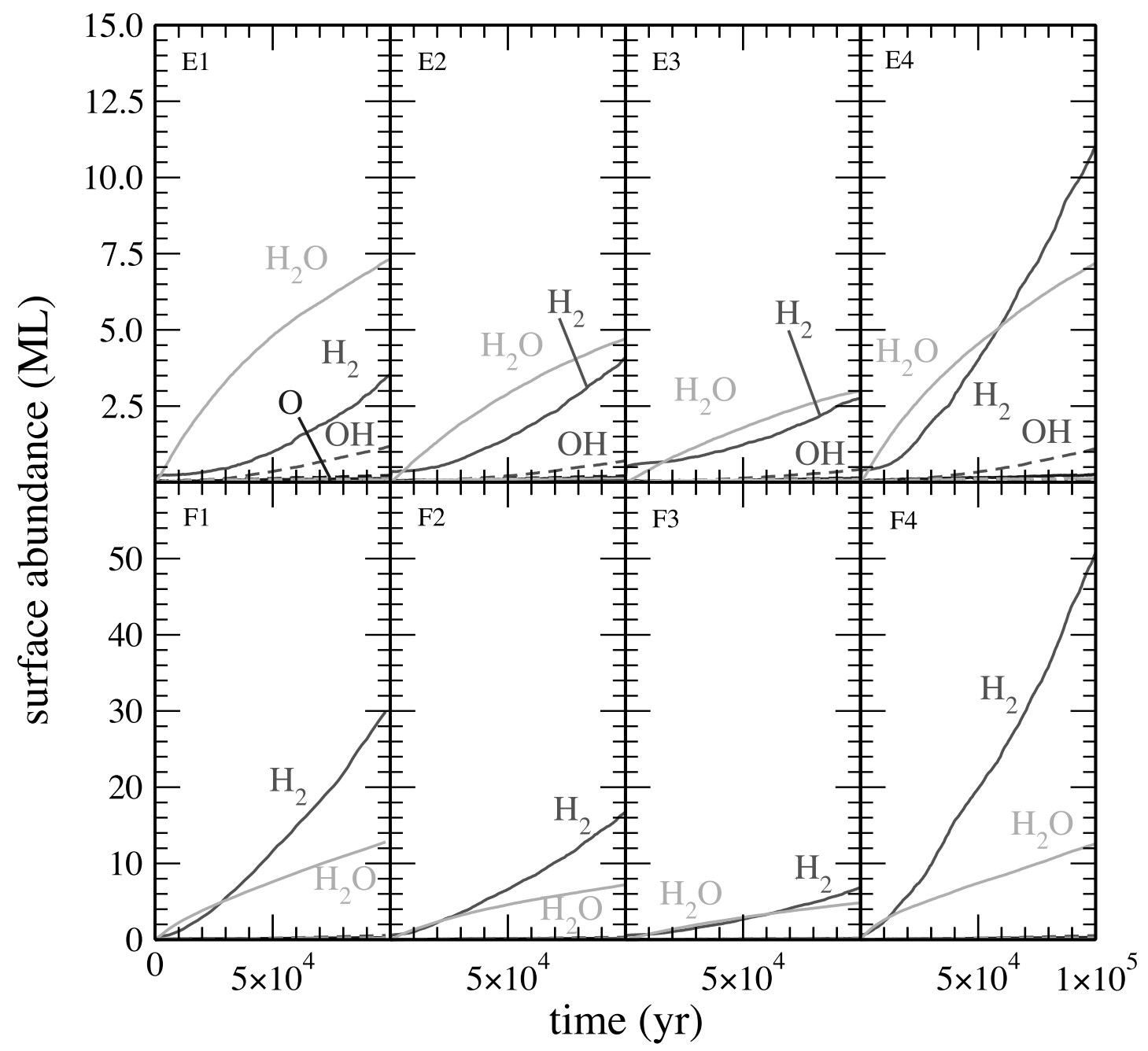

FIG. 8. - Surface abundance with a high hopping barrier-to-desorption energy of 0.78 as a function of time for different conditions in a dense molecular cloud. See Table 4 for physical conditions in each panel. [See the electronic edition of the Journal for a color version of this figure.] 
A2

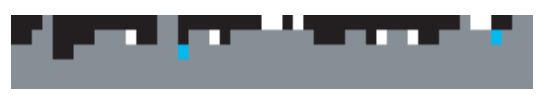

C2

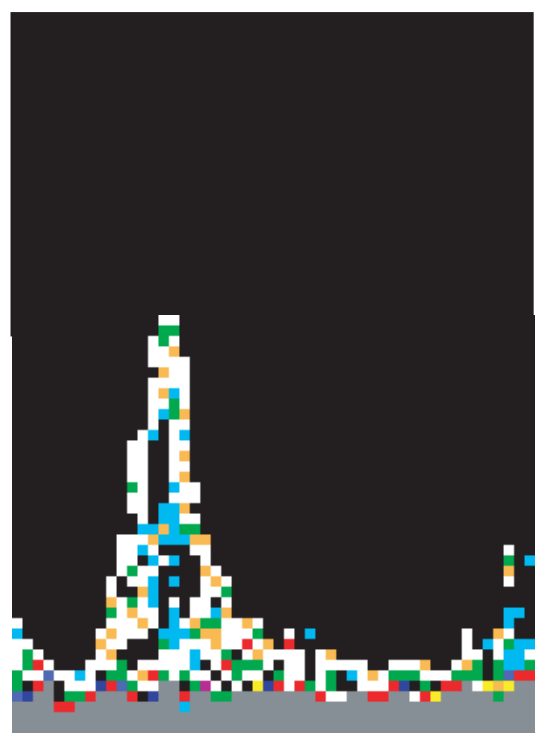

B2

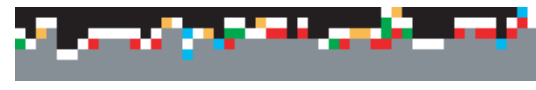

D2

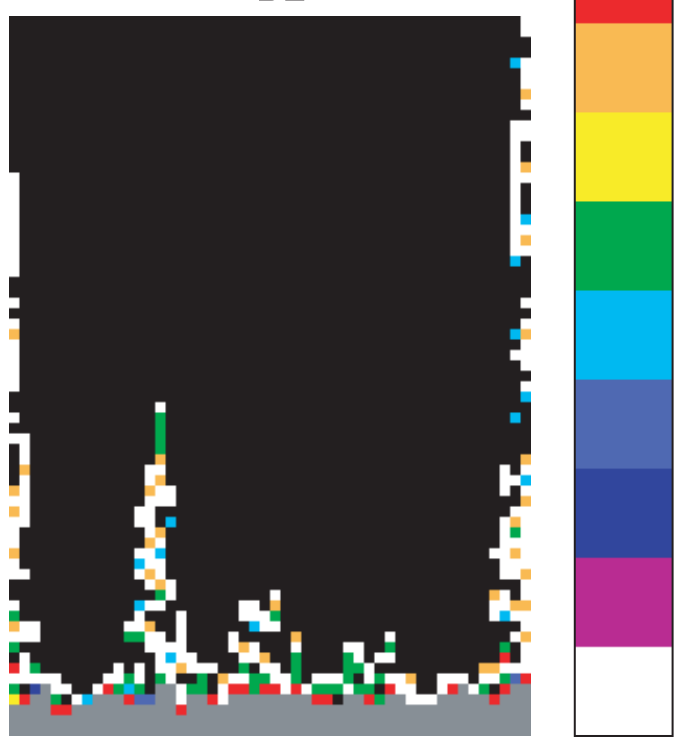

grain

void

0

H

$\mathrm{O}_{2}$

$\mathrm{OH}$

$\mathrm{H}_{2}$

$\mathrm{O}_{2} \mathrm{H}$

$\mathrm{H}_{2} \mathrm{O}_{2}$

$\mathrm{O}_{3}$

$\mathrm{H}_{2} \mathrm{O}$

FIg. 9.- Vertical cross sections of ice mantles after $3 \times 10^{5}$ yr for four different physical conditions in diffuse and translucent clouds (A2, B2, C2, D2; Table 4) obtained with a high hopping barrier-to-desorption energy of 0.78 . The molecules are color coded.
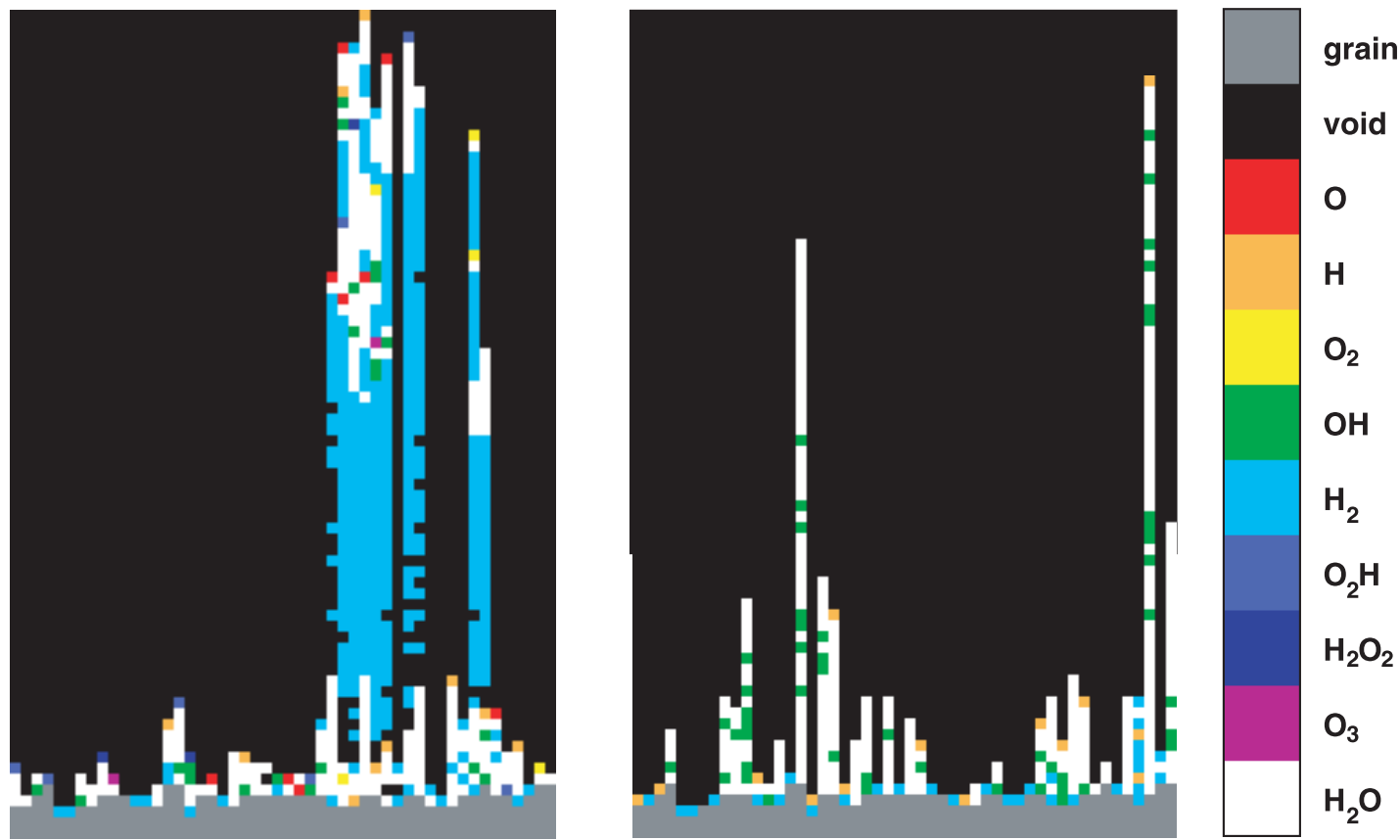

FIG. 10.- Vertical cross sections of ice mantles after $1 \times 10^{5} \mathrm{yr}$ for two different dense-cloud physical conditions obtained with a high hopping barrier-to-desorption energy of 0.78. Left panel (E2): $A_{V}=5, T_{\mathrm{gr}}=12 \mathrm{~K}, T_{\text {gas }}=20 \mathrm{~K}$ and $n_{\mathrm{H}}=5 \times 10^{3} \mathrm{~cm}^{-3}$. Right panel (F2): $A_{V}=10, T_{\mathrm{gr}}=10 \mathrm{~K}, T_{\text {gas }}=10 \mathrm{~K}$, and $n_{\mathrm{H}}=2 \times 10^{4} \mathrm{~cm}^{-3}$. The molecules are color coded. 

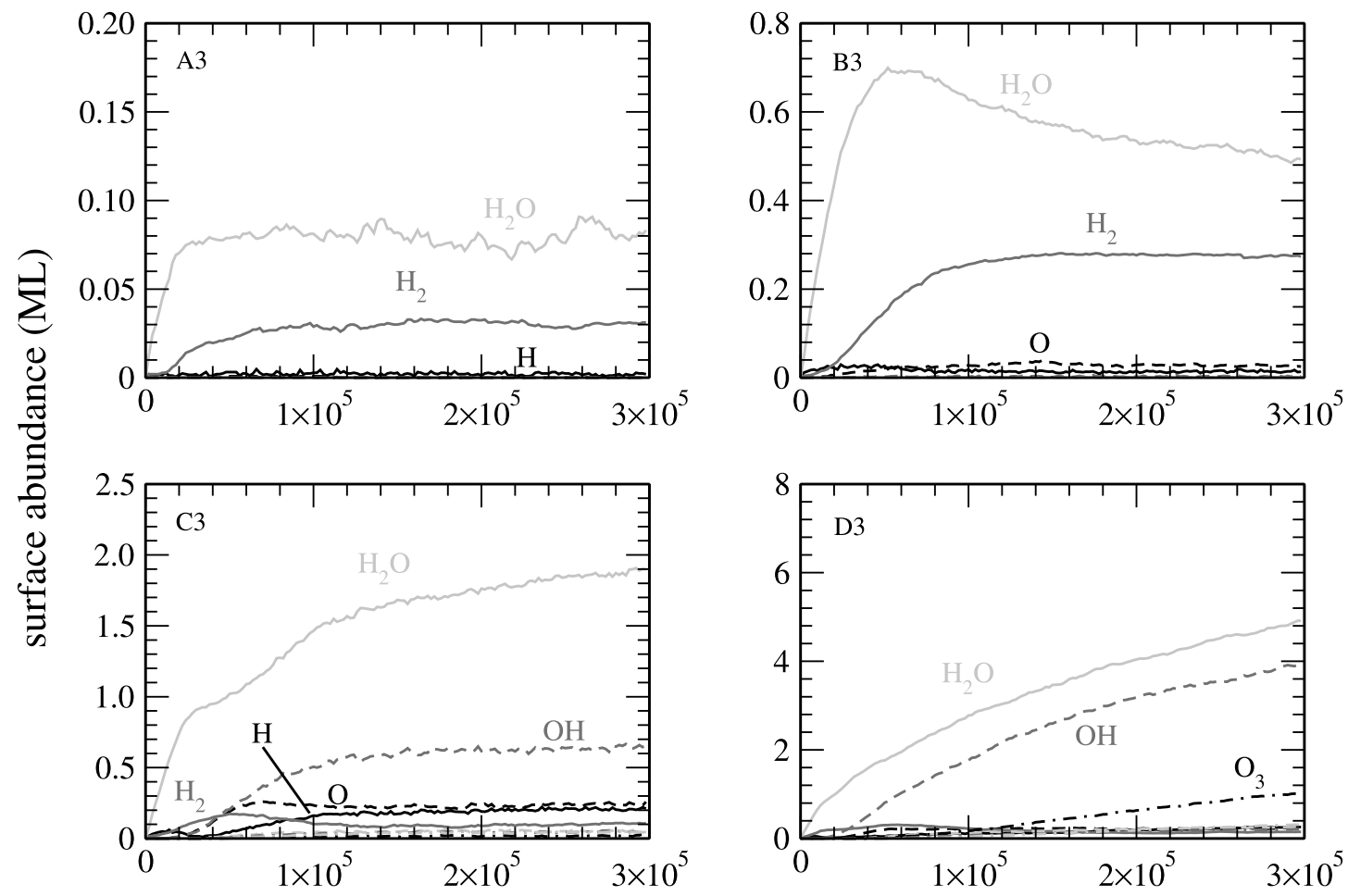

time $(\mathrm{yr})$

FIG. 11.- Surface abundance as a function of time starting with a bare grain with a smooth surface structure. [See the electronic edition of the Journal for a color version of this figure.]

other species change slightly in the very diffuse regions. $\mathrm{A} 3$ has less $\mathrm{H}_{2}$ and $\mathrm{B} 3$ less $\mathrm{O}$ for the smoother surface. In the other two cases, the surface is completely covered with ice, which determines the energetics so that the difference in surface abundances between the smoother and the rougher surfaces becomes negligible.

\section{REFERENCES}

Al-Halabi, A., Kleyn, A., van Dishoeck, E. F., \& Kroes, G. J. 2002, J. Phys. Chem. B, 106, 6515

Al-Halabi, A., \& van Dishoek, E.F. 2007, MNRAS, in press

Allen, M., \& Robinson, G. W. 1975, ApJ, 195, 81

Andersson, S., van Dishoeck, E. F., \& Kroes, G. J. 2006, J. Chem. Phys., 124, 064715

Avgul, N. N., \& Kiselev, A. V. 1970, in Chemistry and Physics of Carbon, ed. P. L. Walker (Vol. 6; New York: Dekker)

Awad, Z., Chigai, T., Kimura, Y., Shalabiea, O. M., \& Yamamoto, T. 2005, ApJ, 626, 262

Ayotte, P., Smith, R. S., Stevenson, K. P., Dohnálek, Z., Kimmel, G. A., \& Kay, B. D. 2001, J. Geophys. Res., 106, 33387

Biham, O., Furman, I., Pirronello, V., \& Vidali, G. 2001, ApJ, 553, 595

Bottinelli, S., et al. 2004, ApJ, 615, 354

Buch, V., \& Czerminski, R. 1991, J. Chem. Phys., 95, 6026

Caselli, P., Hasegawa, T. I., \& Herbst, E. 1993, ApJ, 408, 548

Chang, Q., Cuppen, H. M., \& Herbst, E. 2005, A\&A, 434, 599

Collings, M. P., Anderson, M. A., Chen, R., Dever, J. W., Viti, S., Williams, D. A., \& McCoustra, M. R. S. 2004, MNRAS, 354, 1133

Collings, M. P., Dever, J. W., Fraser, H. J., \& McCoustra, M. R. S. 2003, Ap\&SS, 285, 633

Cuppen, H. M., \& Herbst, E. 2005, MNRAS, 361, 565

Cuppen, H. M., Morata, O., \& Herbst, E. 2006, MNRAS, 367, 1757

Dulieu, F., Amiaud, L., Baouche, S., Momeni, A., Fillion, J.-H., \& Lemaire, J. L. 2005, Chem. Phys. Lett., 404, 187

Fraser, H. J., Collings, M. P., McCoustra, M. R. S., \& Williams, D. A. 2001, MNRAS, 327, 1165

Fuchs, G., et al. 2007, A\&A, submitted

Gale, R. L., \& Beebe, R. A. 1964, J. Phys. Chem., 68, 555

Garrod, R., Park, I. H., Caselli, P., \& Herbst, E. 2006, Discuss. Faraday Soc., 133,51

Garrod, R., Wakelam, V., \& Herbst, E. 2007, A\&A, 467, 1103

Ghio, E., Mattera, L., Salvo, C., Tommasini, F., \& Valbusa, U. 1980, J. Chem. Phys., 73, 556
Han, S. \& Lee, H. M. 2004, Carbon, 42, 2169

Herbst, E., \& Cuppen, H. M. 2006, Proc. Natl. Acad. Sci., 103, 12257

Hiraoka, K., Ohashi, N., Kihara, Y., Yamamoto, K., Sato, T., \& Yamashita, A. 1994, Chem. Phys. Lett., 229, 408

Hollenbach, D., \& Salpeter, E. 1970, J. Chem. Phys., 53, 79

Hornekær, L., Baurichter, A., Petrunin, V. V., Luntz, A. C., Kay, B. D., \& Al-Halabi, A. 2005, J. Chem. Phys., 122, 124701

Jenniskens, P., Blake, D. F., Wilson, M. A., \& Pohorille, A. 1995, ApJ, 455, 389

Katz, N., Furman, I., Biham, O., Pirronello, V., \& Vidali, G. 1999, ApJ, 522, 305

Kimmel, G. A., Dohnálek, Z., Stevenson, K. P., Smith, R. S., \& Kay, B. D. 2001, J. Chem. Phys., 114, 5295

Klemm, R. B., Payne, W. A., \& Stief, L. J. 1975, in Chemical Kinetic Data for the Upper and Lower Atmosphere, ed. S. W. Benson (New York: Wiley), 61

Kroes, G. J., \& Andersson, S. 2006, in Astrochemistry: Recent Successes and Current Challenges, ed. D. C. Lis, G. A. Blake, \& E. Herbst (New York: Cambridge Univ. Press), 427

Lee, J. H., Michael, J. V., Payne, W. A., \& Stief, L. J. 1978, J. Chem. Phys., 69, 350

Melius, C. F., \& Blint, R. J. 1979, Chem. Phys. Lett., 64, 183

Nguyen, T. K., Ruffle, D. P., Herbst, E., \& Williams, D. A. 2002, MNRAS, 329,301

Papoular, R. 2005, MNRAS, 362, 489

Perets, H. B., Biham, O., Manicó, G., Pirronello, V., Roser, J., Swords, S., \& Vidali, G. 2005, ApJ, 627, 850

Picaud, S., Hoang, P. N. M., Hamad, S., Mejias, J. A., \& Lago, S. 2004, J. Phys. Chem. B, 108, 5410

Pirronello, V., Liu, C., Roser, J. E., \& Vidali, G. 1999, A\&A, 344, 681

Pontoppidan, K. M., van Dishoeck, E. F., \& Dartois, E. 2004, A\&A, 426, 925

Prasad, S. S., \& Tarafdar, S. P. 1983, ApJ, 267, 603

Ruffle, D. P., \& Herbst, E. 2001, MNRAS, 322, 770

Sandford, S. A., \& Allamandola, L. J. 1993, ApJ, 409, L65 
Schiff, H. I. 1973, in Physics and Chemistry of Upper Atmospheres, ed. B. M. McCormac (Dordrecht: Reidel), 85

Speedy, R. J., Debenedetti, P. G., Smith, R. S., Huang, C., \& Kay, B. D. 1996, J. Chem. Phys., 105, 240

Tielens, A. G. G. M., \& Allamandola, L. J. 1987, in Interstellar Processes, ed. D. J. Hollenbach \& H. A. Thronson, Jr. (Dordrecht: Kluwer), 397

Tielens, A. G. G. M., \& Hagen, W. 1982, A\&A, 114, 245

Ulbricht, H., Moos, G., \& Hertel, T. 2002, Phys. Rev. B, 66, 075404

van Dishoeck, E., Jonkheid, B., \& van Hemert, M. 2006, Discuss. Faraday Soc., 133, 231
Vidali, G., Ihm, G., Kim, H.-Y., \& Cole, M. W. 1991, Surf. Sci. Rep., 12, 133 Watanabe, N., \& Kouchi, A. 2002, ApJ, 571, L173

Whittet, D. C. B., Gerakines, P. A., Hough, J. H., \& Shenoy, S. S. 2001, ApJ, 547,872

Whittet, D. C. B., et al. 1997, ApJ, 490, 729

1998, ApJ, 498, L159

Williams, D. A., Hartquist, T. W., \& Whittet, D. C. B. 1992, MNRAS, 258, 599 